\title{
The initial stage of transition in pipe flow: role of optimal base-flow distortions
}

\author{
By M. I. GAVARINI, ${ }^{1}$ A. BOTTARO ${ }^{2} \dagger$ \\ AND F. T. M. NIEUWSTADT ${ }^{1} \ddagger$ \\ ${ }^{1}$ J. M. Burgers Centre, Lab. Aero \& Hydrodynamics, Delft University of Technology, \\ 2628 CA Delft, The Netherlands \\ ${ }^{2}$ Institute de Mécanique des Fluides de Toulouse, Allée du Pr. Camille Soula, 31400 Toulouse, France
}

(Received 27 October 2003 and in revised form 24 June 2004)

We explore the spatial growth of disturbances developing on top of a base flow given by the Hagen-Poiseuille profile, which has been modified by a small axisymmetric and axially invariant distortion. Such deviations from the ideal parabolic profile may, for instance, occur in experiments as a result of experimental uncertainties. The optimal distortion (i.e. the distortion with a prescribed norm that induces the maximum growth rate) is computed by a variational technique. Unstable modes are found to exist for very small values of the norm of the deviation at low Reynolds numbers, and the instability is governed by an inviscid mechanism. The growth of these modes and the ensuing transition to turbulence is then studied by means of direct numerical simulations. Two possible paths of transition are found, one based on the exponential amplification of axisymmetric disturbances and the subsequent formation of $\Lambda$-vortices and the other based on the growth and breakdown of streamwise streaks.

\section{Introduction}

In the transition process from laminar to turbulent flow in a cylindrical pipe, at least two stages can be distinguished: the initial and the late stage. The initial stage is related to the receptivity, i.e. the way the flow filters incoming disturbances, and to their initial linear growth into finite-amplitude perturbations, for which eventually nonlinear mechanisms (late stage) become important. Recently, much progress has been made in our understanding of the late stage of transition. Both experiments (Han, Tumin \& Wygnanski 2000) and numerical simulations (Reuter \& Rempfer 2000) have shown that in pipe flow this late stage is accompanied by the formation of $\Lambda$-like vortices characterized by strong shear layers and by spikes in the temporal traces of the velocity. These spikes are associated to ring-like vortices that separate from the tips of the large $\Lambda$-vortices. Similar structures have also been found in the late stages of transition in boundary layers, either in the K-, N- or O-regimes of breakdown (see Berlin, Wiegel \& Henningson 1999). These observations indicate that a considerable degree of similarity exists in the late stage of transition in a pipe and in a boundary layer.

$\dagger$ Present address: DIAM, Università di Genova, via Montallegro 1, 16145 Genova, Italy. $\ddagger$ Author to whom correspondence should be addressed: e-mail f.nieuwstadt@wbmt.tudelft.nl 
The initial stage of transition in a flat-plate boundary layer in the presence of acoustic disturbances in the free stream and of roughness elements on the surface usually starts with the excitation of unstable Tollmien-Schlichting (TS) waves if the Reynolds number exceeds a critical value. When these two-dimensional TS waves reach a sufficiently large amplitude, a three-dimensional secondary instability develops. This instability produces a streamwise component of the vorticity which eventually leads to the formation of $\Lambda$-vortices, i.e. to the start of the late stage described above. However, when the disturbance environment is dominated by reasonably high levels of freestream turbulence, Matsubara \& Alfredsson (2001) have shown that transition closely approaches the oblique transition path (see Berlin, Lundbladh \& Henningson 1994), in which the initial exponential growth is by-passed by another linear and stronger amplification mechanism, i.e. the non-modal growth of streamwise-elongated structures. This is an example of the 'weak-disturbance by-pass' introduced by Morkovin (1993). In the presence of even higher levels of free-stream turbulence, the whole initial stage of linear growth is by-passed and the late stage of transition sets in immediately. This has been categorized as the 'strong-disturbance by-pass' by Morkovin.

For the cylindrical pipe geometry, much less is known about the initial stage of transition, one of the reasons being that there are no linearly unstable modes of the TS kind. Analytical treatment of pipe flow transition, through the classical normalmode approach, has shown that the fully developed parabolic Hagen-Poiseuille profile is linearly stable at all Reynolds numbers, Re (see Lessen, Sadler \& Liu 1968; Davey \& Drazin 1969; Garg \& Rouleau 1972). Although a rigorous proof of stability exists only for axisymmetric disturbances (Herron 1991), there is very strong evidence (e.g. Salwen, Cotton \& Grosch 1980; Meseguer \& Trefethen 2003) that all linear perturbations decay exponentially for all values of $R e$ and for any value of the streamwise and azimuthal wavenumbers $\alpha$ and $m$. Nevertheless, in experiments, transition to turbulence in pipe flow is typically observed for $R e \geqslant R e_{c r_{l}}$. Here, $R e_{c r_{l}}$ is the lower limit of the critical (we should rather say 'transitional') Reynolds number, which means that below $R e_{c r l}$ all finite perturbations decay. The value of $R e_{c r l}$ has been estimated experimentally to lie in the range $1760<R_{c_{r l}}<2300$. We speak in this case of subcritical transition to turbulence, as transition occurs at values of the Reynolds numbers, for which linear theory indicates stability.

On the other hand, in very carefully performed experiments, conceived to exclude all causes for external perturbations, laminar Hagen-Poiseuille flow can be maintained up to very large values of the Reynolds number. In the very first experiments conducted by Reynolds in 1880, for instance, a transitional value of the parameter, that eventually became known as the Reynolds number, close to 13000 was observed. Reynolds' original observations were published three years later (Reynolds 1883) and for a long time his results remained unchallenged and unexplained by theory. In more recent times, Pfenniger (1961) reports $R e_{c r_{u}} \simeq 10^{5}$, where $R e_{c r_{u}}$ denotes the upper limit of the critical Reynolds number. Different values of $R e_{c r_{u}}$ are reported for individual experiments (see, for example, Draad, Kuiken \& Nieuwstadt 1998). For all these experiments, however, it turns out that $R e_{c r_{u}}>R e_{d e v}$, where $R e_{d e v}$ is the value of the Reynolds number, for which the velocity profile at the end of the pipe deviates by less than $1 \%$ from the theoretical Hagen-Poiseuille profile. This could imply that the transition process found in these experiments may not be considered as natural transition of cylindrical pipe flow, but rather as the result of the instabilities of the developing boundary layer on the wall of the pipe. On the other hand, these high values of $R e_{c r_{u}}$ do not contradict the estimate $R e_{c r} \rightarrow \infty$ of linear stability theory for the fully developed flow. 
Another route to transition in pipe flow has been reported in the experiments of Darbyshire \& Mullin (1995); Eliahou, Tumin \& Wygnanski (1998); Draad et al. (1998) and the numerical simulations of Ma et al. (1999) and Reuter \& Rempfer (2000). In this case, transition is forced by the introduction of finite disturbances at the wall, in particular by periodic blowing and suction through slots in the pipe wall. Although the perturbations are finite, they are nevertheless small and, without additional amplification, they should decay as predicted by the classical linear eigen-analysis. However, it has been suggested by various authors (Boberg \& Brosa 1988; Bergström 1992, 1993; Trefethen et al. 1993; Schmid \& Henningson 1994) that, in this case, the non-normality of the linearized Navier-Stokes operator can play an important role, since it implies potential for strong initial amplification of disturbances, also denoted as transient growth. As a result of this growth, the disturbance amplitude may reach a level for which nonlinear interactions are triggered and the eventual viscous decay predicted by the linear asymptotic analysis is overcome. The scenario of amplification of small finite perturbations through transient growth in pipe flow was, for instance, suggested by Ma et al. (1999) and O'Sullivan \& Breuer (1994a, $b$ ) based on numerical simulations. Adopting the definitions that were introduced above for a boundarylayer flow, this transition scenario could be referred to as by-pass transition, since the linear amplification of disturbances requires a by-pass of the single mode growth. The estimates for $R e_{c r_{l}}$ that have been obtained following this by-pass scenario lie in the range of $1760<R e_{c r_{l}}<2300$, which has already been mentioned and this provides an encouraging argument for the transient-growth picture. It should also be noted that these values still differ strongly from the lower bound for transition, i.e. $R e_{c_{e}}=81.49$, obtained through the energy method (Joseph \& Carmi 1969; Schmid \& Henningson 1994).

The non-normality of the linearized operator has another important consequence, i.e. the high sensitivity of some of its eigenvalues to perturbations, such as, for example, distortions in the base flow (Bottaro, Corbett \& Luchini 2003). Such a strong sensitivity is clearly observed in the experiments by Darbyshire \& Mullin (1995), who show a broad intermittent range of turbulent flow and decaying perturbations in the disturbance amplitude versus Reynolds-number space. In the carefully controlled experiments by Eliahou et al. (1998), it was noted that, by exciting the azimuthal periodic modes $m= \pm 2$ with time-periodic suction and blowing through slots in the pipe wall, transition could be triggered at $R e=2200$ only for sufficiently largedisturbance amplitudes. Eliahou et al. (1998) concluded with the suggestion that 'transition to turbulence in a fully developed pipe Poiseuille flow can occur only after the parabolic velocity profile became distorted.'

Base-flow deviations from the theoretical parabolic Hagen-Poiseuille profile can be ascribed to a variety of physical reasons. They are likely to occur in any experimental set-up owing to surface-roughness effects, external volume forces, inflow inhomogeneities, and so on. Even when the theoretical profile is linearly stable, as is the case for the Hagen-Poiseuille flow, it is possible that small base-flow distortions render the flow linearly unstable. This possible scenario of instability has already been examined by Gill (1965) in an effort to explain some laboratory measurements.

The objective of the present work is to show by means of theory and numerical simulations that the sensitivity of the eigenmodes to small distortions in the base flow can be a cause for initial exponential growth of perturbations. It is expected that those eigenvalues, which show the highest sensitivity to infinitesimal variations in the base flow, are the most affected and can become unstable. These so-called most 'receptive' eigenvalues have been recently identified by Tumin (1996) through a 
spatial analysis which employs the direct and adjoint stability operator. Our analysis here differs from that of Tumin because, given these most 'receptive' eigenvalues, we identify so-called 'optimal' base-flow deviations, i.e. those distortions from the Hagen-Poiseuille profile, which maximize the instability of the flow in a way to be described shortly. It will be shown that deviations with a very small (but finite) norm are already sufficient for the modified flow to become exponentially unstable at low Reynolds numbers. This exponential instability, which can also act in combination with transient-growth mechanisms, may amplify disturbances to a level where eventually nonlinear interactions can take over bringing the flow to the late stage of transition.

In the next two sections, we describe the theory of the optimally perturbed base flow. First, a method based on spatial eigen-analysis is presented to determine the sensitivity of individual eigenvalues to variations introduced in the base flow. Then a standard variational technique is used to determine the optimal base-flow deviations, i.e. those deviations that can best destabilize the flow. Finally, in $\S 4$, we describe results from direct simulations of the nonlinear governing equations carried out to study the evolution of unstable eigenmodes, and discuss the viability of the transition scenario identified.

\section{The theory}

As the starting point of our analysis we introduce a cylindrical coordinate system $(x, r, \theta)$. Here, $x, r$ and $\theta$ denote the axial, radial and azimuthal directions, respectively, and $u, v$ and $w$ the corresponding velocity components. The laminar solution to the incompressible Navier-Stokes equations for a cylindrical pipe geometry is the wellknown Hagen-Poiseuille flow, which in non-dimensional form, using the centreline velocity $U_{\max }$ and the pipe radius $R$ as scales, reads:

$$
\boldsymbol{V}(r)=U(r) \boldsymbol{e}_{x}=\left(1-r^{2}\right) \boldsymbol{e}_{x}, \quad P(x)=P_{0}-\frac{4}{R e} x,
$$

where $P_{0}$ is the static pressure at $x=0$ and $\boldsymbol{e}_{x}$ is the unit vector in the $x$-direction. The Reynolds number is $R e=U_{\max } R / v$. Linearization of the full Navier-Stokes equation around the laminar solution $U$ produces:

$$
\begin{gathered}
\frac{\partial u}{\partial x}+\frac{1}{r} \frac{\partial(r v)}{\partial r}+\frac{1}{r} \frac{\partial w}{\partial \theta}=0, \\
\frac{\partial u}{\partial t}+U \frac{\partial u}{\partial x}+v \frac{\mathrm{d} U}{\mathrm{~d} r}=-\frac{\partial p}{\partial x}+\frac{1}{R e} \nabla^{2} u, \\
\frac{\partial v}{\partial t}+U \frac{\partial v}{\partial x}=-\frac{\partial p}{\partial r}+\frac{1}{R e}\left(\nabla^{2} v-\frac{v}{r^{2}}-\frac{2}{r^{2}} \frac{\partial w}{\partial \theta}\right), \\
\frac{\partial w}{\partial t}+U \frac{\partial w}{\partial x}=-\frac{1}{r} \frac{\partial p}{\partial \theta}+\frac{1}{R e}\left(\nabla^{2} w-\frac{w}{r^{2}}+\frac{2}{r^{2}} \frac{\partial v}{\partial \theta}\right),
\end{gathered}
$$

with

$$
\nabla^{2}=\frac{\partial^{2}}{\partial x^{2}}+\frac{1}{r} \frac{\partial}{\partial r}\left(r \frac{\partial}{\partial r}\right)+\frac{1}{r^{2}} \frac{\partial^{2}}{\partial \theta^{2}} .
$$

Next, we assume the disturbance quantities $\boldsymbol{v}=(u, v, w)$ and $p$ to be of the form:

$$
\begin{aligned}
& \boldsymbol{v}(r, \theta, x, t)=\widehat{\boldsymbol{v}}(r, x ; m, \omega) \mathrm{e}^{\mathrm{i}(m \theta-\omega t)}, \\
& p(r, \theta, x, t)=\widehat{p}(r, x ; m, \omega) \mathrm{e}^{\mathrm{i}(m \theta-\omega t)},
\end{aligned}
$$


where $m$ is the azimuthal wavenumber, $\omega$ the dimensionless circular frequency and $\widehat{\boldsymbol{v}}=(\widehat{u}, \widehat{v}, \widehat{w})$ and $\widehat{p}$ the complex disturbance amplitudes. Following Tumin (1996), two auxiliary variables are introduced, namely $\widehat{v}_{x}=\partial \widehat{v} / \partial x$ and $\widehat{w}_{x}=\partial \widehat{w} / \partial x$. The equations $(2.2 a)-(2.2 d)$ can then be rewritten as a system of first-order differential equations in $x$, for which the vector of the unknowns is given by $\boldsymbol{a}(x, r ; m, \omega, R e)=$ $\left(\widehat{v}, \widehat{v}_{x}, \widehat{u}, \widehat{p}, \widehat{w}, \widehat{w}_{x}\right)$. The resulting system in compact notation reads:

$$
\frac{\partial \boldsymbol{a}}{\partial x}=\mathscr{C}_{0} \boldsymbol{a}+\mathscr{C}_{1} \frac{\partial \boldsymbol{a}}{\partial r}+\mathscr{C}_{2} \frac{\partial^{2} \boldsymbol{a}}{\partial r^{2}},
$$

where $\mathscr{C}_{k}(k=0,1,2)$ are $6 \times 6$ coefficient matrices whose non-zero entries are given in Appendix A.

In a spatial stability analysis, the frequency $\omega$ is a real parameter and the solution to system (2.4) is assumed to behave as:

$$
\boldsymbol{a}(x, r ; m, \omega, \boldsymbol{R e})=\boldsymbol{a}_{\alpha}(r ; m, \omega, \boldsymbol{R} e) \mathrm{e}^{\mathrm{i} \alpha x} .
$$

Introduction into (2.4) results in the following eigenvalue problem:

$$
\mathrm{i} \alpha \boldsymbol{a}_{\alpha}=\mathscr{L} \boldsymbol{a}_{\alpha},
$$

where $\alpha$ is the complex eigenvalue, whose real and imaginary parts give, respectively, the wavenumber and the growth rate of the eigenmode in the streamwise direction. The linear operator $\mathscr{L}$ is given by:

$$
\mathscr{L}=\sum_{k=0}^{2} \mathscr{C}_{k} \frac{\mathrm{d}^{k}}{\mathrm{~d} r^{k}} .
$$

At the pipe wall, the no-slip boundary condition is assumed:

$$
r=1: a_{1} \equiv \widehat{v}=0, \quad a_{3} \equiv \widehat{u}=0, \quad a_{5} \equiv \widehat{w}=0
$$

and, as a result, the other variables must satisfy the conditions:

$$
r=1: a_{4}^{\prime} \equiv p^{\prime}=\frac{1}{R e} a_{1}^{\prime \prime}, \quad a_{2} \equiv \widehat{v}_{x}=0, \quad a_{6} \equiv \widehat{w}_{x}=0,
$$

in which primes indicate derivation with respect to the radial coordinate.

Although $r=0$ is not a physical boundary, it is a numerical boundary and the condition that must be imposed at the pipe centreline is that all flow quantities remain bounded. This is accomplished by the following conditions for $r \rightarrow 0$, as a function of the azimuthal wavenumber $m$ :

$$
\begin{gathered}
m=0: a_{1}=a_{3}^{\prime}=a_{5}=0, \Rightarrow a_{2}=a_{4}^{\prime}=a_{6}=0, \\
m= \pm 1: a_{1}^{\prime}=a_{3}=a_{5}^{\prime}=0, \Rightarrow a_{2}^{\prime}=a_{4}=a_{6}^{\prime}=0, \\
|m|>1: a_{1}=a_{3}=a_{5}=0, \Rightarrow a_{2}=a_{4}=a_{6}=0 .
\end{gathered}
$$

The numerical solution of the resulting eigenvalue problem is achieved by the use of a Chebyshev pseudospectral collocation technique. To determine the adequate number $(N+1)$ of Chebyshev polynomials, spectra obtained with $N=64$ and $N=128$ collocation points have been compared. With $N=64$, the first 60 eigenvalues, taken in order of increasing absolute value of their imaginary part, have been found to be sufficiently well resolved. When it was necessary to investigate higher eigenvalues in the spectrum, 128 collocation points have been used.

As an example, in figure 1, the spectrum of eigenvalues for azimuthal index $m=1$, Reynolds number $R e=3000$ and frequency $\omega=0.5$ is displayed. As mentioned in 


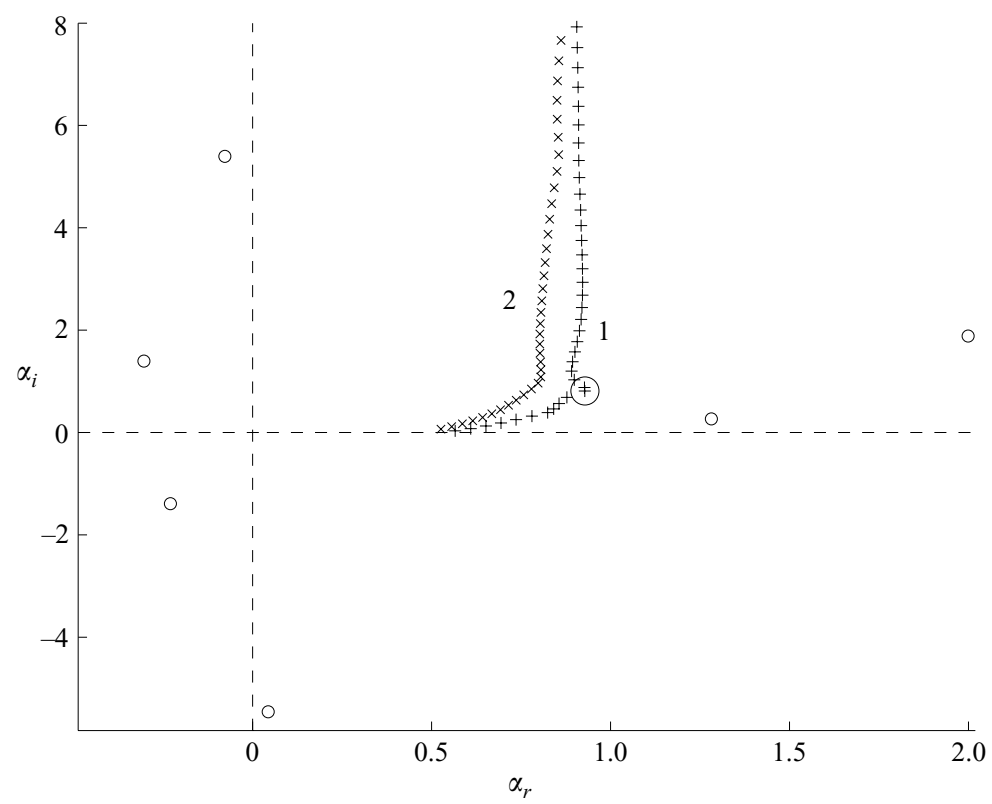

Figure 1. Spatial spectrum of eigenvalues for Hagen-Poiseuille flow at $R e=3000, m=1$, $\omega=0.5$. The large circle includes the two most receptive eigenvalues (cf. $\S 3.1$.) The numbers 1 and 2 denote the two main branches of eigenvalues (represented respectively by + and $\times$, to distinguish them from the rest of the spectrum, $\bigcirc$ ).

$\S 1$, all available evidence indicates that linear modes are stable. The presence of some eigenvalues with $\alpha_{i}<0$ does not imply instability; these modes are simply propagating upstream, reflecting the fact that the initial-value problem for spatially developing disturbances is not well posed. The property of upstream and downstream propagation of some of these modes has been recently verified in the numerical simulations by Ma et al. (1999). In figure 1, for the purpose of subsequent data presentation, we have labelled with 1 and 2 the two neighbouring branches belonging to the two main groups of eigenvalues and we have indicated them with different symbols.

\section{Optimal base-flow deviations}

\subsection{The sensitivity function $G_{U}$}

Let us introduce an infinitesimal real variation $\delta U$ in the base-flow velocity profile $U$ and compute the corresponding variation in the eigenvalues $\delta \alpha$ and eigenfunctions $\delta \boldsymbol{a}_{\alpha}$ of system (2.5). The variational system reads:

$$
\mathrm{i} \delta \alpha \boldsymbol{a}_{\alpha}+\mathrm{i} \alpha \delta \boldsymbol{a}_{\alpha}=\delta \mathscr{L} \boldsymbol{a}_{\alpha}+\mathscr{L} \delta \boldsymbol{a}_{\alpha},
$$

where $\delta \mathscr{L}$ is given in matrix form by:

$$
\delta \mathscr{L}=\left[\begin{array}{cccccc}
0 & 0 & 0 & 0 & 0 & 0 \\
0 & \operatorname{Re} \delta U & 0 & 0 & 0 & 0 \\
0 & 0 & 0 & 0 & 0 & 0 \\
\left(\frac{\delta U}{r}-\delta U^{\prime}\right) & 0 & 0 & 0 & \frac{\mathrm{i} m \delta U}{r} & 0 \\
0 & 0 & 0 & 0 & 0 & 0 \\
0 & 0 & 0 & 0 & 0 & \operatorname{Re} \delta U
\end{array}\right]+\left[\begin{array}{ccc}
0 & \cdots & 0 \\
0 & \cdots & 0 \\
0 & \cdots & 0 \\
\delta U & \cdots & 0 \\
0 & \cdots & 0 \\
0 & \cdots & 0
\end{array}\right] \frac{\mathrm{d}}{\mathrm{d} r} .
$$


In the analysis that follows, we will make use of a scalar product between two vectors $\boldsymbol{f}$ and $\boldsymbol{g}$, defined as:

$$
(\boldsymbol{f}, \boldsymbol{g}) \equiv \int_{0}^{1} r \boldsymbol{g}^{\dagger} \boldsymbol{f} \mathrm{d} r=\int_{0}^{1} r\left(g_{1}^{*} f_{1}+g_{2}^{*} f_{2}+\cdots+g_{6}^{*} f_{6}\right) \mathrm{d} r,
$$

where the superscripts $\dagger$ and $*$ denote, respectively, the conjugate transpose and the complex conjugate. Upon taking the inner product of both sides of the variational system (3.1) with a function $\boldsymbol{b}_{\alpha}$ :

$$
\left(\mathrm{i} \delta \alpha \boldsymbol{a}_{\alpha}, \boldsymbol{b}_{\alpha}\right)+\left(\mathrm{i} \alpha \delta \boldsymbol{a}_{\alpha}, \boldsymbol{b}_{\alpha}\right)=\left(\delta \mathscr{L} \boldsymbol{a}_{\alpha}, \boldsymbol{b}_{\alpha}\right)+\left(\mathscr{L} \delta \boldsymbol{a}_{\alpha}, \boldsymbol{b}_{\alpha}\right),
$$

and after some manipulations, including the integration by parts of the term $\delta U^{\prime}$ in $\delta \mathscr{L},(3.2)$ can be reduced to the form:

$$
\mathrm{i} \delta \alpha \int_{0}^{1} r \boldsymbol{b}_{\alpha}^{\dagger} \boldsymbol{a}_{\alpha} \mathrm{d} r+\left(\delta \boldsymbol{a}_{\alpha},-\mathrm{i} \alpha^{*} \boldsymbol{b}_{\alpha}\right)=\mathrm{i} \int_{0}^{1} r G_{U} \delta U \mathrm{~d} r+\left(\delta \boldsymbol{a}_{\alpha}, \mathscr{L}_{a} \boldsymbol{b}_{\alpha}\right),
$$

with $G_{U}$ given by:

$$
G_{U}=-\mathrm{i} \operatorname{Re} b_{2}^{*} a_{2}+b_{4}^{*}\left[\frac{m a_{5}-2 \mathrm{i} a_{1}}{r}-2 \mathrm{i} a_{1}^{\prime}\right]-\mathrm{i} a_{4}^{\prime *} a_{1}-\mathrm{i} \operatorname{Re} b_{6}^{*} a_{6} .
$$

The function $\boldsymbol{b}_{\alpha}$ is now chosen to be a solution of the adjoint eigenvalue problem, defined by

$$
-\mathrm{i} \alpha^{*} \boldsymbol{b}_{\alpha}=\mathscr{L}_{a} \boldsymbol{b}_{\alpha}=\sum_{k=0}^{2}(-1)^{k} \frac{1}{r} \frac{\mathrm{d}^{k}\left(r \mathscr{C}_{k}^{\dagger} \boldsymbol{b}_{\alpha}\right)}{\mathrm{d} r^{k}},
$$

with the following boundary conditions for $r=1$ :

$$
b_{2}=b_{4}=b_{6}=0 \Rightarrow b_{2}^{\prime}=b_{5}=0, b_{1}+\frac{1}{R e} b_{4}^{\prime}=0
$$

and for $r \rightarrow 0$

$$
\begin{aligned}
& m=0: b_{2}=b_{4}^{\prime}=b_{6}=0 \Rightarrow b_{1}=b_{5}=0,-\frac{3}{2} b_{2}^{\prime \prime}+b_{3}^{\prime}=0 ; \\
& m= \pm 1: b_{2}^{\prime}=b_{4}=b_{6}^{\prime}=0, \Rightarrow b_{3}=0, b_{1}+\mathrm{i} m b_{5}=0, b_{5}^{\prime}+\frac{\mathrm{i} m}{2 R e} b_{4}^{\prime \prime}=0 ; \\
& |m|>1: b_{2}=b_{4}=b_{6}=0 \Rightarrow b_{3}=0, b_{1}+\frac{1}{R e} b_{4}^{\prime}=0, b_{5}+\frac{\mathrm{i} m}{R e} b_{4}^{\prime}=0 .
\end{aligned}
$$

The functions $\boldsymbol{b}_{\alpha}$ and $\boldsymbol{a}_{\alpha}$ are normalized so that the bi-orthonormality condition is satisfied:

$$
\left(\boldsymbol{a}_{\alpha}, \boldsymbol{b}_{\beta}\right)=\int_{0}^{1} r \boldsymbol{b}_{\beta}^{\dagger} \boldsymbol{a}_{\alpha} \mathrm{d} r=\delta_{\alpha \beta} .
$$

With this choice for $\boldsymbol{b}_{\alpha}$, the variation $\delta \alpha$ in the eigenvalue can be evaluated from (3.2) as:

$$
\delta \alpha=\int_{0}^{1} r G_{U} \delta U \mathrm{~d} r
$$

where $G_{U}$ represents the sensitivity function of each eigenvalue $\alpha$ to base-flow variations.

Relation (3.4) shows that $\left(r G_{U}\right)$ can be interpreted as an integrating factor between a variation introduced in the base flow $\delta U$ and the corresponding variation in the eigenvalue $\delta \alpha$. Depending on the property of $G_{U}$, it is possible that small base-flow 


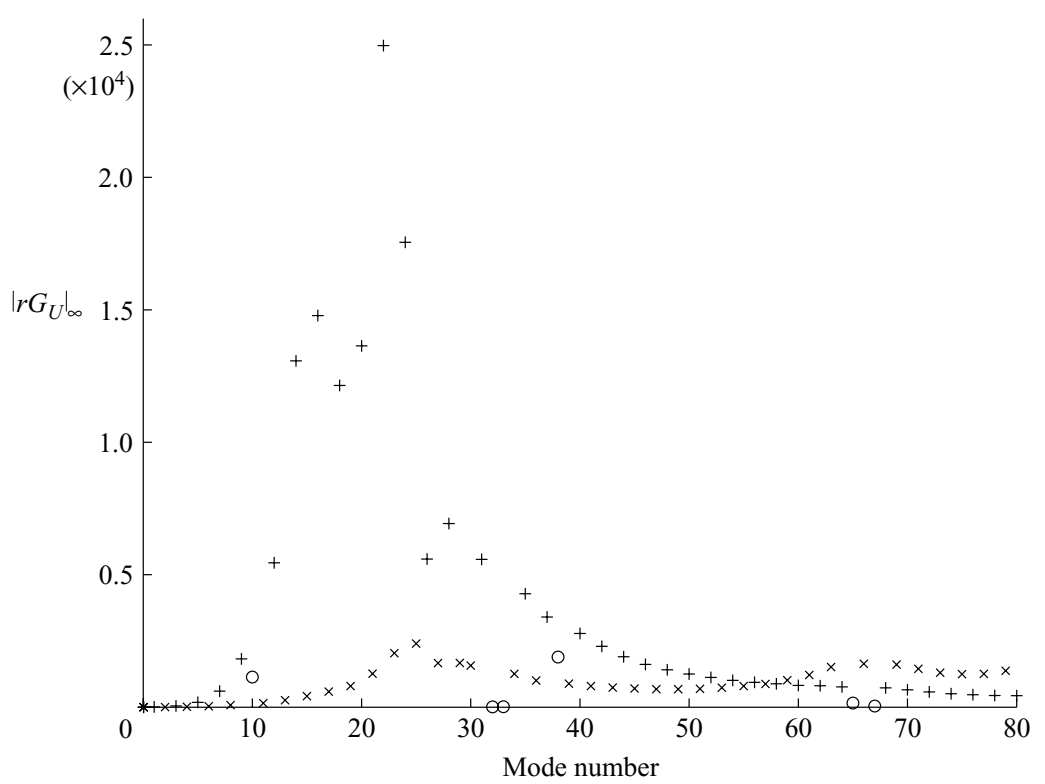

FIGURE 2. Infinity norm of $\left(r G_{U}\right)$ for the eigenvalues shown in figure 1 , numbered in order of increasing $\left|\alpha_{i}\right|$.

deviations render the flow linearly unstable. Our interest is therefore primarily focused on those eigenvalues, for which the sensitivity function $G_{U}$ is large. By solving the direct and adjoint problem, the function $G_{U}$ has been computed for all eigenvalues of the spectrum, according to the definition (3.3). The results for the infinity norm, i.e. the largest absolute value, of $\left(r G_{U}\right)$ are presented in figure 2 for the first 80 eigenvalues of the spectrum shown in figure 1 . Here, the symbols correspond to those used in figure 1 to denote the eigenvalue branches.

As can be seen from figure 2, the least stable eigenvalue has a very small infinity norm of the sensitivity function, while the maximum values for $G_{U}$ are attained by the eigenmodes numbered 22, for which $\alpha=0.9279+0.8111$ i, and 24 , for which $\alpha=0.9272+0.8791 \mathrm{i}$ (the corresponding eigenvalues are encircled in figure 1 ). It also appears that the modes corresponding to the first family of eigenvalues (indicated by a ' + ' sign) are more sensitive than those belonging to the second family, at least up to mode 58. The higher sensitivity found for the modes belonging to the first family, and in particular for modes 22 and 24, agrees with the result reported by Tumin (1996), who indicates that precisely those modes are most receptive to periodic suction and blowing through a slot at the wall.

Figure 3 illustrates the absolute value of $\left(r G_{U}\right)$ as a function of the radial direction for these two most sensitive eigenmodes and also for the least stable eigenmode. The sensitivity functions for modes 22 and 24 have a maximum close to the wall (around $r=0.66$ ) and this maximum is about three orders of magnitude larger than the maximum value of the least stable mode, which is located closer to the centreline.

\subsection{The 'optimally' perturbed base flow}

Our interest now shifts to finding 'optimal' deviations from the ideal base flow, which in our case is the Hagen-Poiseuille flow and which will be called $U_{\text {ref }}$ from now on. 'Optimal' in this case means base-flow distortions which lead to exponentially growing perturbations, but at the same time minimize the deviation from $U_{r e f}$. Equivalently, 


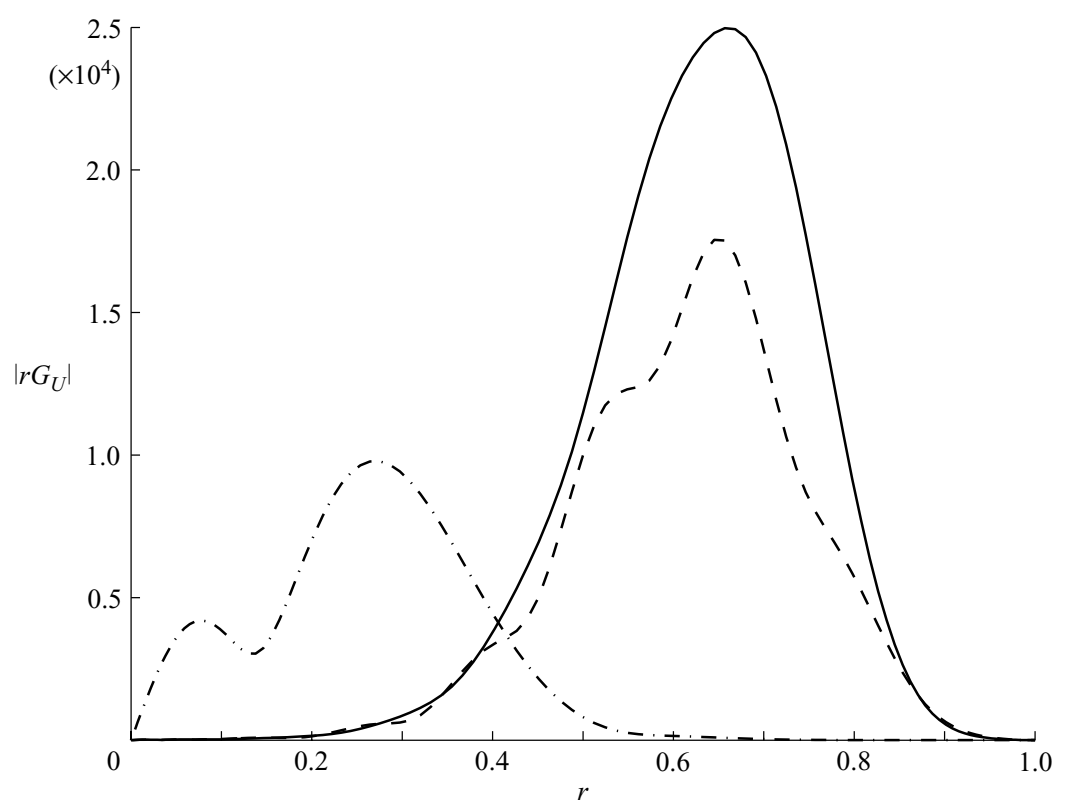

Figure 3. Absolute value of $\left(r G_{U}\right)$ for mode 22 (solid line), mode 24 (dashed line) and mode 1 multiplied by $10^{3}$ (dash-dotted line).

our goal can be stated as the minimization of the imaginary part of the eigenvalue $\alpha_{i}$ of a downstream propagating mode (so that it is displaced to the lower half-plane) by satisfying a constraint on the magnitude of the velocity deviation, $\Delta U=U-U_{\text {ref }}$, expressed by the norm:

$$
\int_{0}^{1} r \Delta U^{2} \mathrm{~d} r=\epsilon
$$

Note that $\epsilon$ is, in general, taken to be small, but it does not need to be infinitesimally small. The resulting constrained minimization problem can be reduced to an unconstrained one, by introducing the Lagrange multiplier $\lambda$. This results in the minimization of the functional:

$$
\mathscr{I}=\alpha_{i}-\lambda\left[\int_{0}^{1} r\left(U-U_{r e f}\right)^{2} \mathrm{~d} r-\epsilon\right],
$$

a necessary condition of which is given by:

$$
\delta \mathscr{I}=\delta \alpha_{i}-2 \lambda \int_{0}^{1} r \Delta U \delta U \mathrm{~d} r=0 .
$$

On the basis of (3.4), the equation above results in the following optimality condition:

$$
\delta \alpha_{i}=2 \lambda \int_{0}^{1} r \Delta U \delta U \mathrm{~d} r=\int_{0}^{1} r G_{U_{i}} \delta U \mathrm{~d} r
$$

where $G_{U_{i}}$ is the imaginary part of the sensitivity function. Since (3.6) must hold for arbitrary variations $\delta U$, it must be that:

$$
\Delta U=\frac{G_{U_{i}}}{2 \lambda}
$$




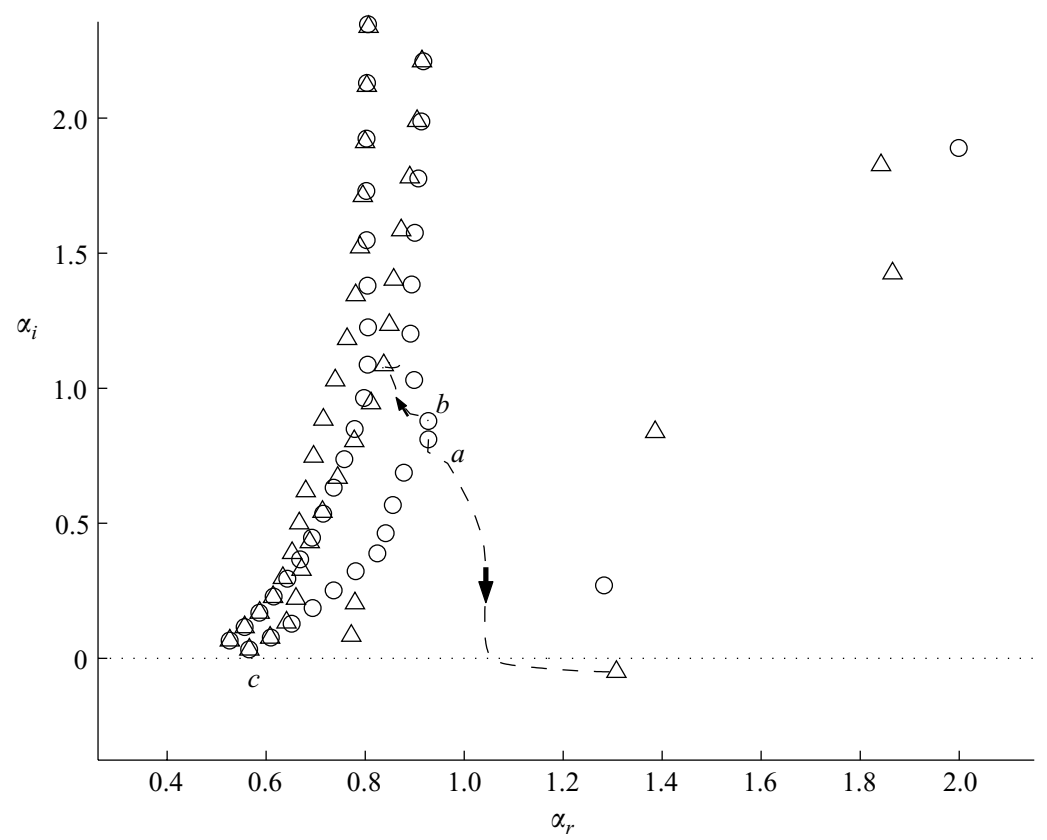

FIGURE 4. Spectrum of eigenvalues for Hagen-Poiseuille flow at $R e=3000, m=1, \omega=0.5$ (circles), and for the optimally perturbed base flow with norm $\epsilon=2.5 \times 10^{-5}$, which minimizes $\alpha_{i}$ of mode 22 labelled with $a$ (triangles). The paths with the arrow indicate the trajectories followed by modes $a$ and $b$ in $\alpha$-space in the course of the iterations using (3.8).

By enforcing (3.5), $\lambda$ is found to be equal to:

$$
\lambda= \pm\left[\frac{1}{4 \epsilon} \int_{0}^{1} r G_{U_{i}}^{2} \mathrm{~d} r\right]^{1 / 2}
$$

where the minus sign corresponds to a minimization.

Since $G_{U_{i}}$ depends on the base flow $U$, (3.7) must be solved iteratively, i.e. by employing the algorithm:

$$
U^{(n+1)}=U^{(n)}-k\left[U^{(n)}-U_{r e f}-\frac{G_{U_{i}}^{(n)}}{2 \lambda^{(n)}}\right],
$$

with superscript $(n)$ denoting the iteration number and $k, 0<k \leqslant 1$, a relaxation parameter. We assume that convergence is reached when $\int_{0}^{1} r\left(U^{(n+1)}-U^{(n)}\right)^{2} \mathrm{~d} r$ is close to zero to machine (double) precision.

With the iterative procedure outlined above, we can compute the 'optimal' base-flow deviation for any eigenvalue $\alpha$. In figure 4 , the spectrum of the eigenvalues for a disturbance with $m=1$ and $\omega=0.5$, superimposed to the Hagen-Poiseuille flow at $R e=3000$, is shown again in somewhat more detail. The most receptive eigenvalues have been labelled $a$ and $b$, while the eigenvalue labelled $c$ is the least stable. In the same figure, we have plotted, by using open triangles, the spectrum for the optimally perturbed base flow with norm $\epsilon=2.5 \times 10^{-5}$. As can be seen, a base-flow deviation, even of this very small norm, but specifically targeted to minimize $\alpha_{i}$ of mode $a$, is able to make this eigenvalue cross the real axis to the unstable $\alpha$-plane. At the same time, mode $b$ moves upwards, i.e. it becomes more stable, whereas mode $c$ is 


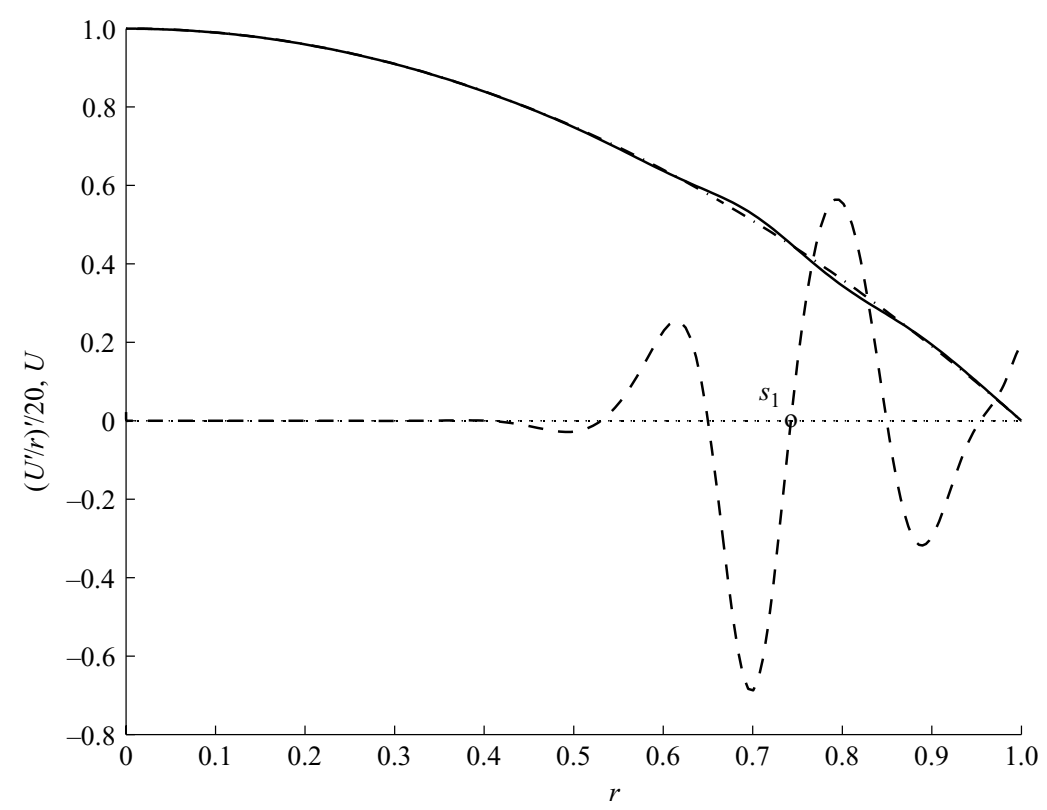

FiguRE 5. Hagen-Poiseuille flow (dash-dotted line) plotted along with the optimally perturbed base flow $U$ which minimizes $\alpha_{i}$ of mode 22 (solid line) and the derivative of the circulation $U^{\prime} / r$ normalized by a factor 20 (dashed line).

negligibly affected. The resulting unstable base flow is plotted in figure 5 together with the reference Hagen-Poiseuille flow and with the function $\left(U^{\prime} / r\right)^{\prime}$, which will be discussed later on. The final unstable eigenvalue is equal to $1.3077-0.0499$ i. Exactly the same value is achieved when the minimization procedure uses mode 24 as starting point (labelled $b$ in figure 4), suggesting the presence of a strong 'attractor' for the highly sensitive modes.

Although we can give no formal proof, we argue that we have found a global minimum of $\alpha_{i}$ for $\epsilon=2.5 \times 10^{-5}$ because, when the procedure starts from a number of other modes as initial condition, it never manages to produce a lower value of $\alpha_{i}$. For example, when starting from the least stable mode (labelled $c$ in figure 4) the local minimum is found to be $\alpha=0.5650+0.0126 \mathrm{i}$, i.e. this mode remains stable. Moreover, in this case, the whole spectrum changes only slightly, as illustrated by figure 6. The corresponding optimally perturbed base flow is plotted in figure 7. As expected from the shape of the sensitivity function for this eigenmode (shown in figure 3), the deviation from ideal pipe Poiseuille flow is now most pronounced close to the axis.

We conclude, therefore, that for the current parameter settings, $m=1, \omega=0.5$, $R e=3000$, the most destabilizing mean flow deviations are localized around $r \simeq 0.66$, as shown in figure 5. This agrees qualitatively with the results obtained by Gill (1965), according to whom, at high Reynolds numbers, base-flow deviations localized around $r \simeq 0.7$ are most effective in destabilizing (axisymmetric) periodic disturbances.

As shown in figure 5, the optimal base-flow deviation produces five points for which $\left(U^{\prime} / r\right)^{\prime}$ crosses the real axis, which is Rayleigh's necessary condition of inviscid instability to axisymmetric disturbances for a flow in a cylindrical geometry (see Batchelor \& Gill 1962). The point denoted by $s_{1}$ satisfies also the modified Fjørtoft's 


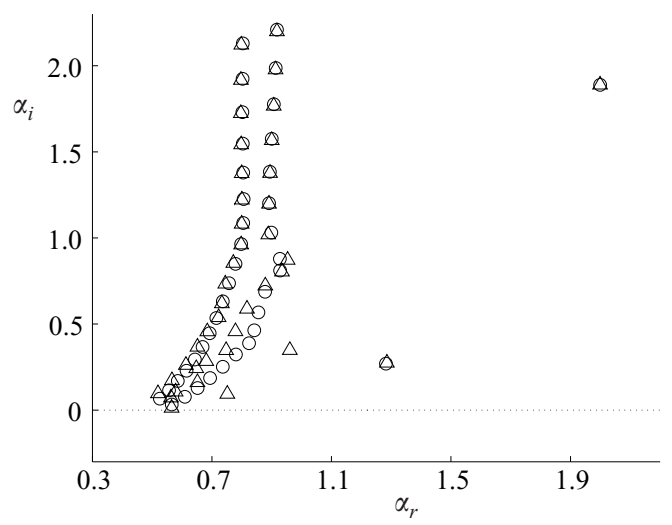

FIGURE 6. Spectrum of eigenvalues for Hagen-Poiseuille flow at $R e=3000, m=1, \omega=0.5$ (circles), and for the optimally perturbed base flow, which minimizes $\alpha_{i}$ of the least stable mode (triangles).

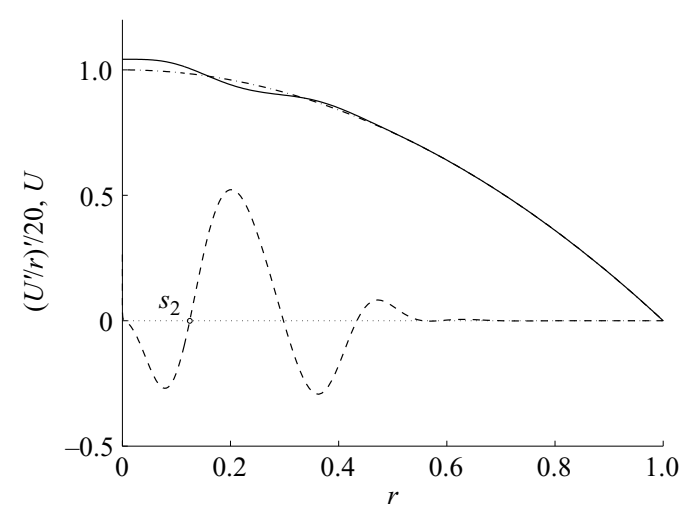

FIGURE 7. Hagen-Poiseuille flow (dash-dotted line) plotted along with the optimally perturbed base flow (solid line), which minimizes $\alpha_{i}$ of the least stable mode. The function $\left(U^{\prime} / r\right)^{\prime}$, normalized by a factor 20 , is also plotted with a dashed line.

criterion:

$$
\left(\frac{U^{\prime}}{r}\right)^{\prime}\left(U-U_{s}\right)<0
$$

with $U_{s}$ the velocity at the radial position corresponding to $s_{1}$ (both the Rayleigh and the Fjørtoft stability criteria are briefly reviewed in Appendix B). This suggests that the instability process of the perturbed base flow may be related to the well-known inflection-point mechanism. However, both criteria mentioned above are not sufficient to guarantee instability. This is borne out by point $s_{2}$ in figure 7 , for which both the Rayleigh and Fjørtoft criteria are satisfied, but the flow is nonetheless stable. A supporting argument to the inflectional nature of the instability is given by the good agreement between the phase speed of the unstable waves in all cases analysed, and the streamwise velocity at the inflection point.

Note that in both figures 5 and 7 , the $r$-derivative of the circulation, i.e. the vorticity divided by the radius, has been scaled by a factor 20 . This confirms the result of Gill (1965) that the actual change produced in the circulation by the base-flow distortion 


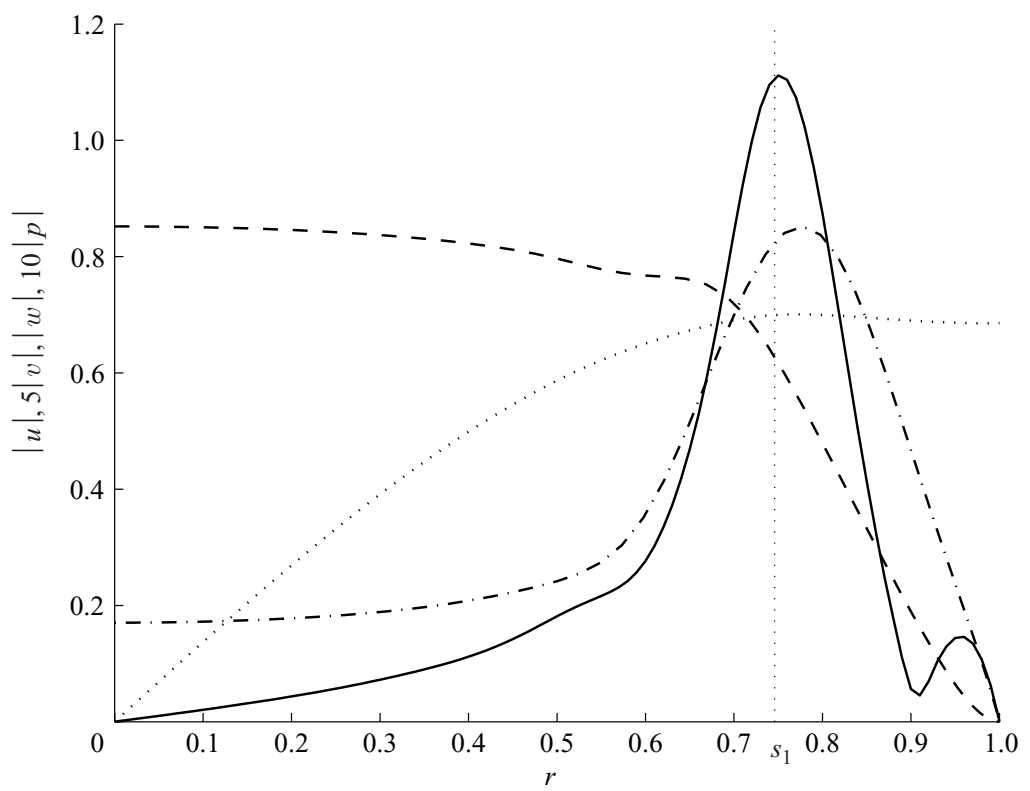

FIGURE 8. Modulus of the eigenfunctions of the unstable mode for the optimally perturbed base flow, which minimizes $\alpha_{i}$ of mode 22; $R e=3000, m=1, \omega=0.5, \epsilon=2.5 \times 10^{-5} \cdot|u|$ (solid line), $5 \times|v|$ (dashed line), $|w|$ (dash-dotted line), $10 \times|p|$ (dotted line).

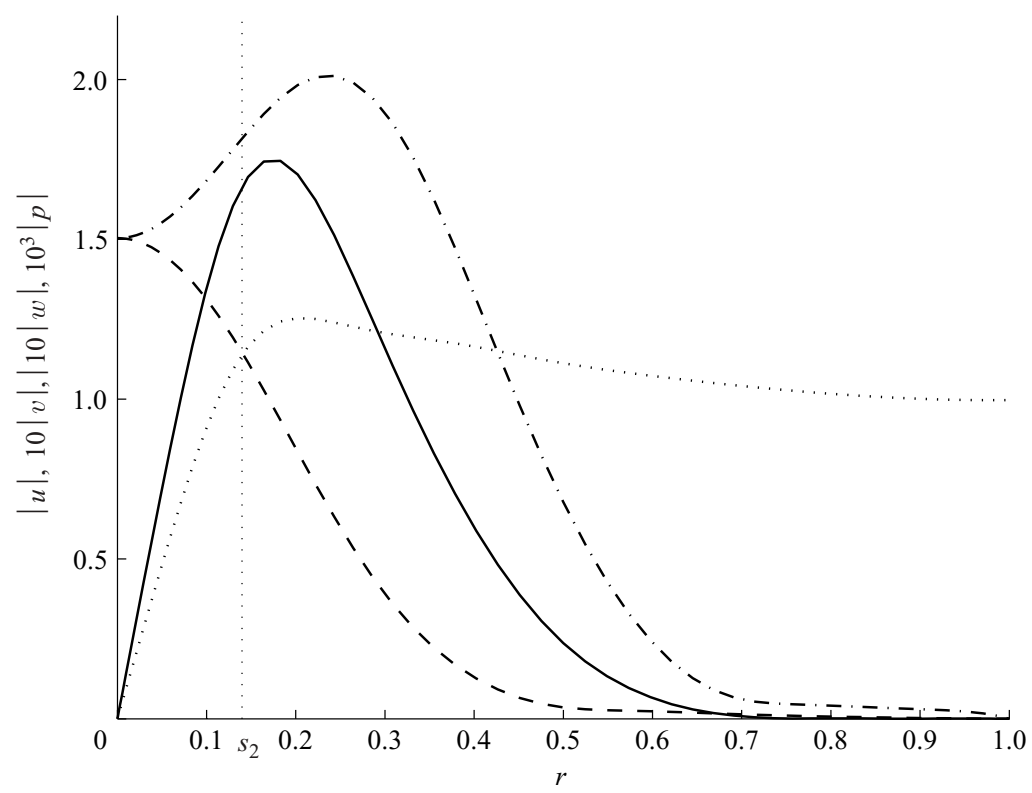

FigurE 9. Modulus of the eigenfunctions of the least stable mode for the optimally perturbed base flow, which minimizes $\alpha_{i}$ of mode 1 (i.e. the least stable eigenvalue); $R e=3000, m=1$, $\omega=0.5, \epsilon=2.5 \times 10^{-5}$. Line styles are the same as in figure 8 .

need only be small, but the gradient of the circulation must be finite to induce a linear instability in the flow.

The eigenfunctions corresponding to the unstable mode of figure 4 and to the least stable mode of figure 6 are displayed in figures 8 and 9. In both cases, we find 
that a peak in axial velocity $u$ occurs close to the points $s_{1}$ and $s_{2}$ where $\left(U^{\prime} / r\right)^{\prime}$ vanishes.

It could be argued that these newly obtained base flows, as shown in figures 5 and 7, are not solutions of the unforced Navier-Stokes equations. However, they are solutions if, for example, a small volume force $f_{x}=-\nabla^{2}(\Delta U) / R e$ or alternatively a small pressure deviation (equal to $-f_{x} x$ ) are applied in the axial direction. In practice, several mechanisms could act as external forcing, allowing for solutions to the Navier-Stokes equations different from the Hagen-Poiseuille flow, at least locally. A few examples are the Coriolis force that Draad et al. (1998) had to deal with in their experiments, convection due to (small) temperature differences between the fluid in the pipe and the outside ambient, or again roughness elements or local disturbances in the geometry, such as curvature due to small bends along the pipe or a slightly non-circular pipe cross-section (see, for instance the work of Davey (1978) and Kerswell \& Davey (1996) on nearly circular elliptical pipes). We can also consider non-stationary phenomena that may occur when, for instance, the pressure gradient changes as a function of time so that the mean flow deviates from the parabolic profile for some time. If, as a consequence of any of these mechanisms, the modified flow can be established and can persist over a sufficiently long axial distance, transition can be triggered by the exponential instability.

The results discussed above can be interpreted as a possible initial stage in the transition process in cylindrical pipe flow. A slightly imperfect base flow allows exponential growth of small disturbances. When these disturbances are allowed to become large enough, they may trigger the nonlinear interactions characteristic of the final stage of transition. We will return to this possible scenario for transition in $\S 4$ when we discuss the numerical simulations.

\subsection{Dependence on $R e, \omega, m$ and $\epsilon$}

In the previous section, we have considered the optimal base-flow deviation only for a fixed setting of parameters. Let us now consider the effect of varying these parameters.

In figure 10 , the growth rate, $-\alpha_{i}$, is plotted as a function of the frequency $\omega$ for a perturbation with $m=1$ and for different values of the Reynolds number. Each point in the curve represents the growth rate of the most sensitive mode for a base flow, which (optimally) deviates from the Hagen-Poiseuille profile by a function $\Delta U$ of norm $\epsilon=10^{-5}$. Clearly, a larger band of frequencies becomes unstable as $R e$ increases. For the chosen values of $m$ and $\epsilon$ the exponential amplification of one eigenmode starts already at $R e$ somewhat below 1760 with $\alpha_{r} \simeq 4$ and $\omega \simeq 3.5$. In figure 11 , the corresponding values of the phase velocity, $c_{p}=\omega / \alpha_{r}$, are displayed. The waves are dispersive and their speed of propagation increases with $\omega$. Furthermore, as $\omega$ increases or the Reynolds number decreases, the phase velocity tends to a value around one, i.e. it approaches the centreline velocity. This is a consequence of the corresponding shift of the optimal base-flow distortion towards the centreline, as can be observed in figure 12, and is related to the fact that the phase speed of the unstable Rayleigh wave is approximately equal to the streamwise velocity at the inflection point.

In figure 13(a), the neutral curve is displayed in the plane $(R e, \omega)$ for the optimally perturbed base-flow profiles with a norm of the base-flow deviation equal to $\epsilon=10^{-5}$. In the same figure, we also show the critical points $\left(R e_{c}, \omega_{c}\right)$, evaluated for different values of $\epsilon$. Clearly, for increasing $\epsilon$, the critical conditions shift to lower values of $R e$. In figure 13(b), the same neutral curve and corresponding symbols are plotted 


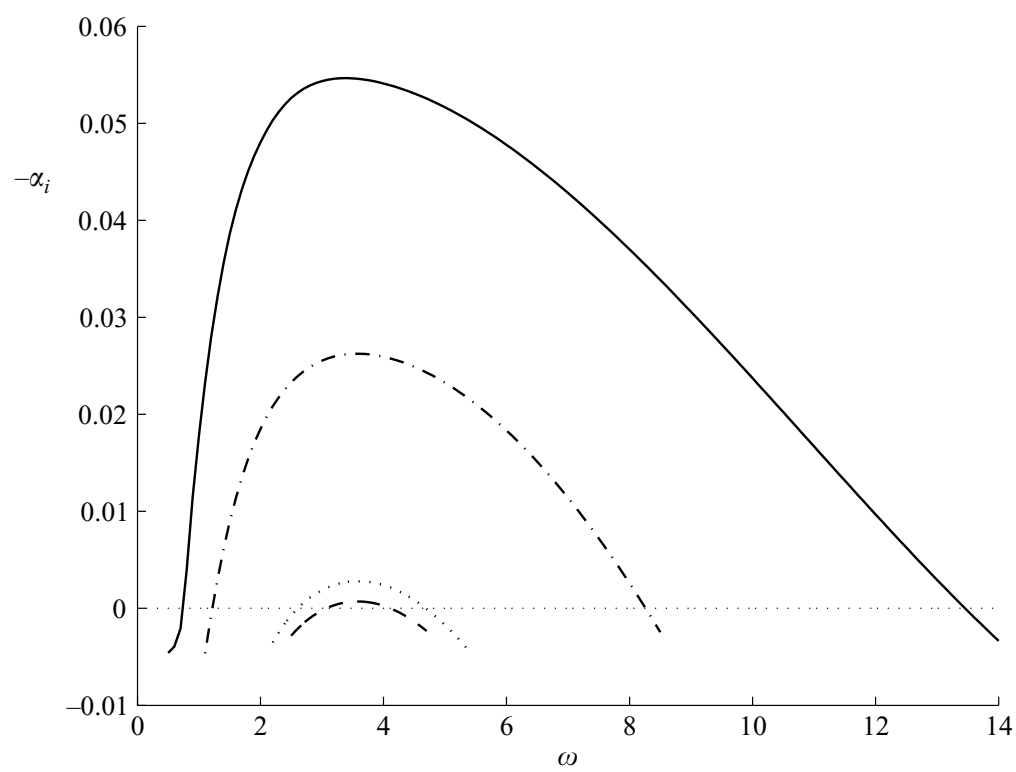

FIGURE 10. Growth rate as a function of frequency for $m=1, \epsilon=10^{-5}, R e=1760$ (dashed line), $R e=1800$ (dotted line), $R e=2300$ (dash-dotted line), $R e=3000$ (solid line).

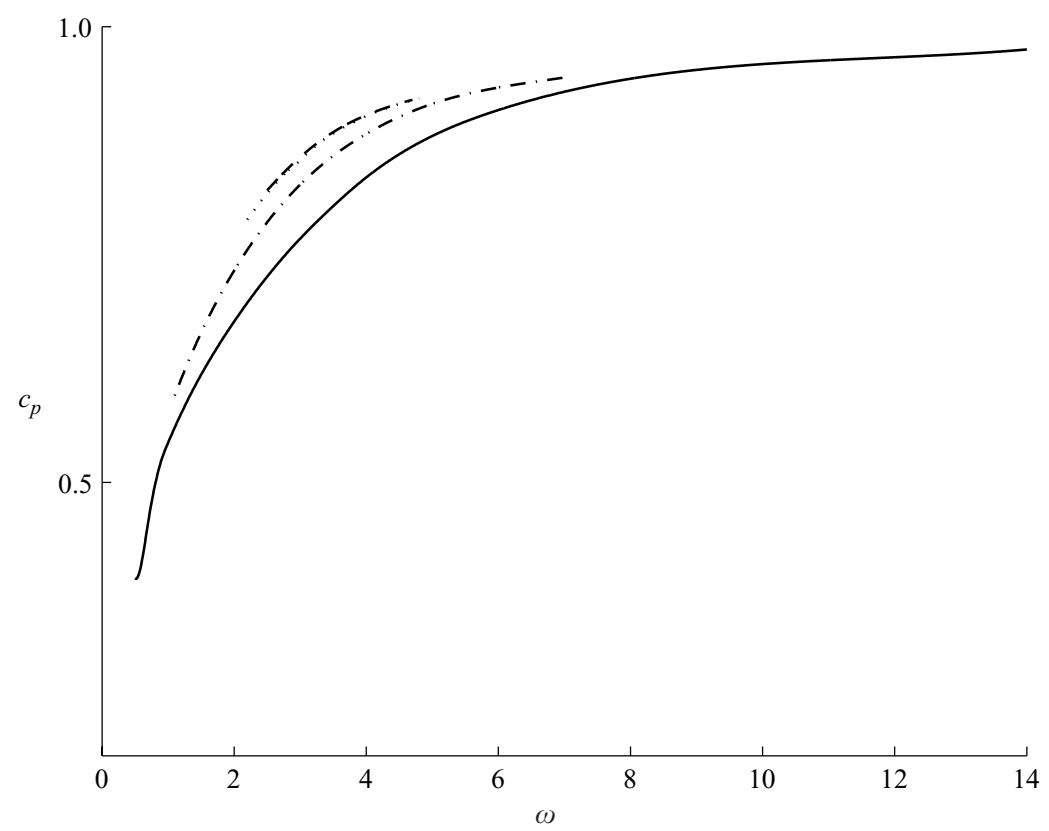

FIGURE 11. Phase velocity as a function of frequency for $m=1, \epsilon=10^{-5}, R e=1760$ (dashed line), $R e=1800$ (dotted line), $R e=2300$ (dash-dotted line), $R e=3000$ (solid line).

in the plane $\left(R e, \alpha_{r}\right)$, from which we can deduce that streamwise waves of shorter wavelengths (and higher frequencies) are preferentially excited when $\epsilon$ decreases.

In figure $14(a)$, the relation between the critical Reynolds number and the norm of the base-flow deviation $\epsilon$, as defined in (3.5), is shown for the case $m=1$. The data 

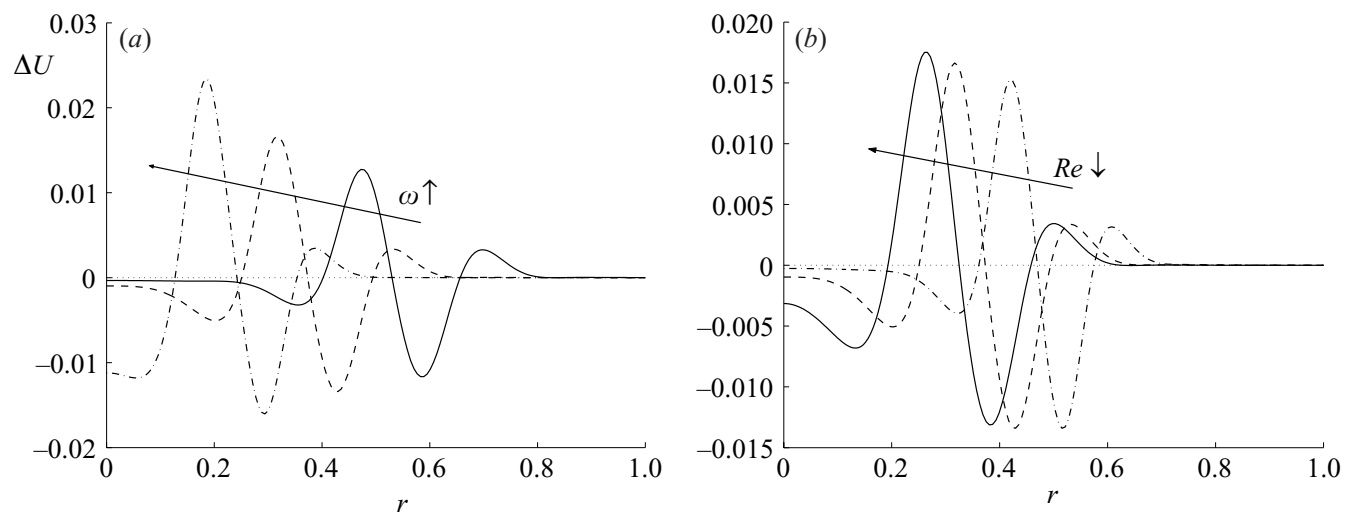

FIGURE 12. Optimal base-flow deviations for $m=1, \epsilon=10^{-5}$. (a) $R e=2300 ; \omega=1.6$ (solid line), $\omega=3.2$ (dashed line), $\omega=6$ (dash-dotted line). (b) $\omega=3.2 ; R e=1800$ (solid line), $R e=2300$ (dashed line), $R e=3000$ (dash-dotted line).
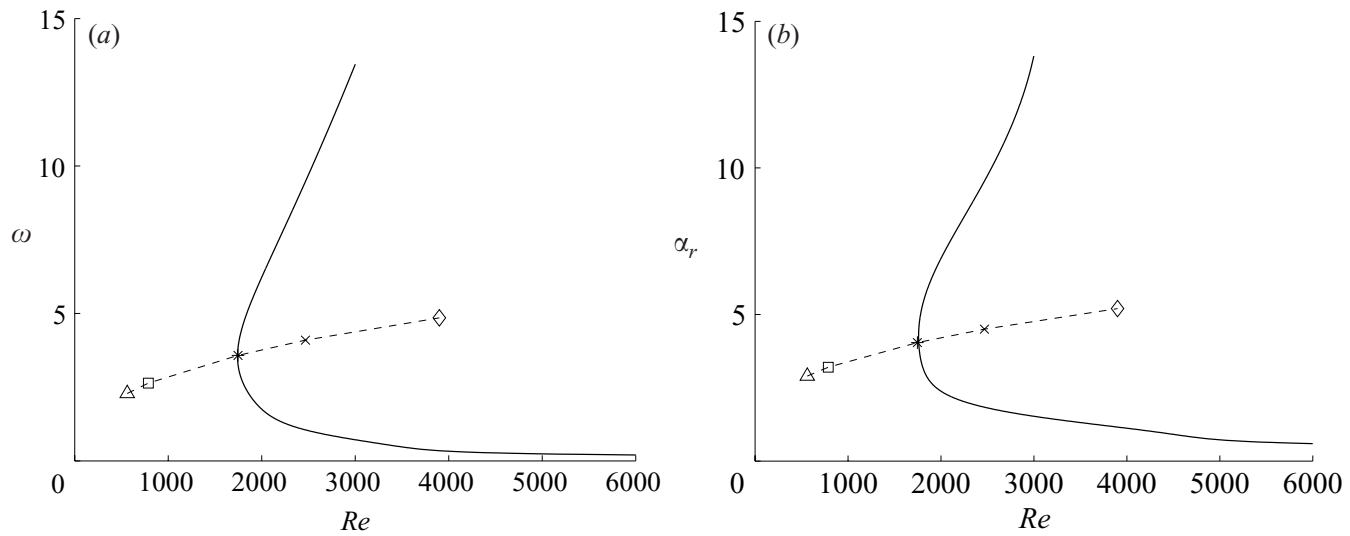

Figure 13. (a) Neutral curve in the $(R e, \omega)$-plane and $(b)$ in the $\left(R e, \alpha_{r}\right)$-plane for $m=1$ and $\epsilon=10^{-5}$ (solid line); symbols give respectively $\left(R e_{c}, \omega_{c}\right)$ and $\left(R e_{c}, \alpha_{r c}\right)$ for different values of $\epsilon: \triangle, \epsilon=10^{-4} ; \square, \epsilon=5 \times 10^{-5} ; *, \epsilon=10^{-5} ; \times, \epsilon=5 \times 10^{-6} ; \diamond, \epsilon=2 \times 10^{-6}$.
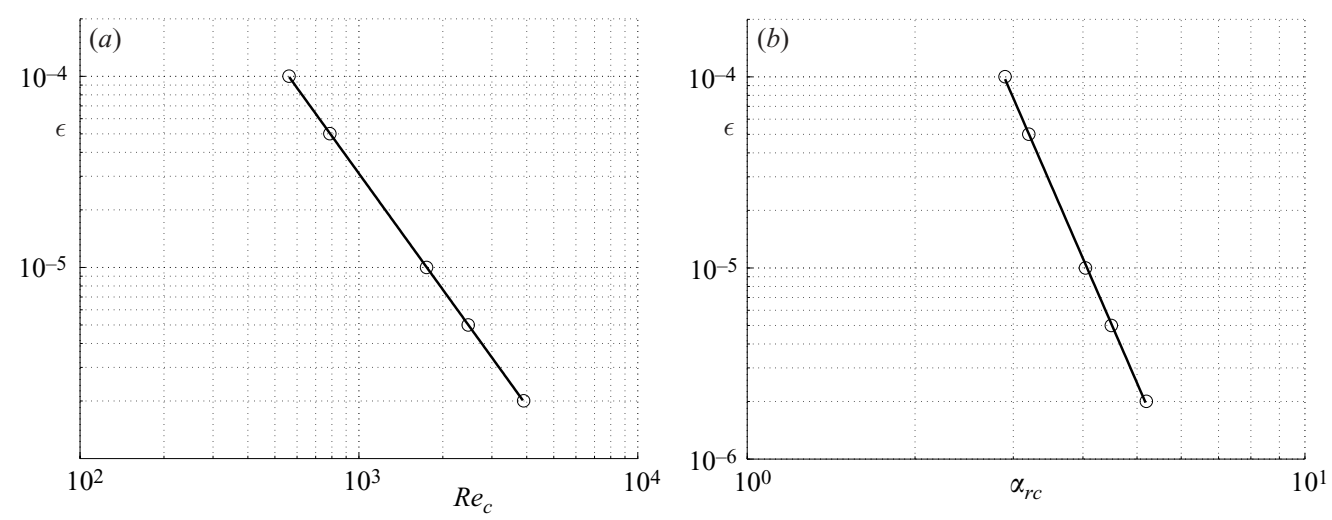

FiguRE 14. Norm of the base-flow deviation as a function of the critical Reynolds number (a) and of the critical streamwise wavenumber $(b)$, for $m=1$. 

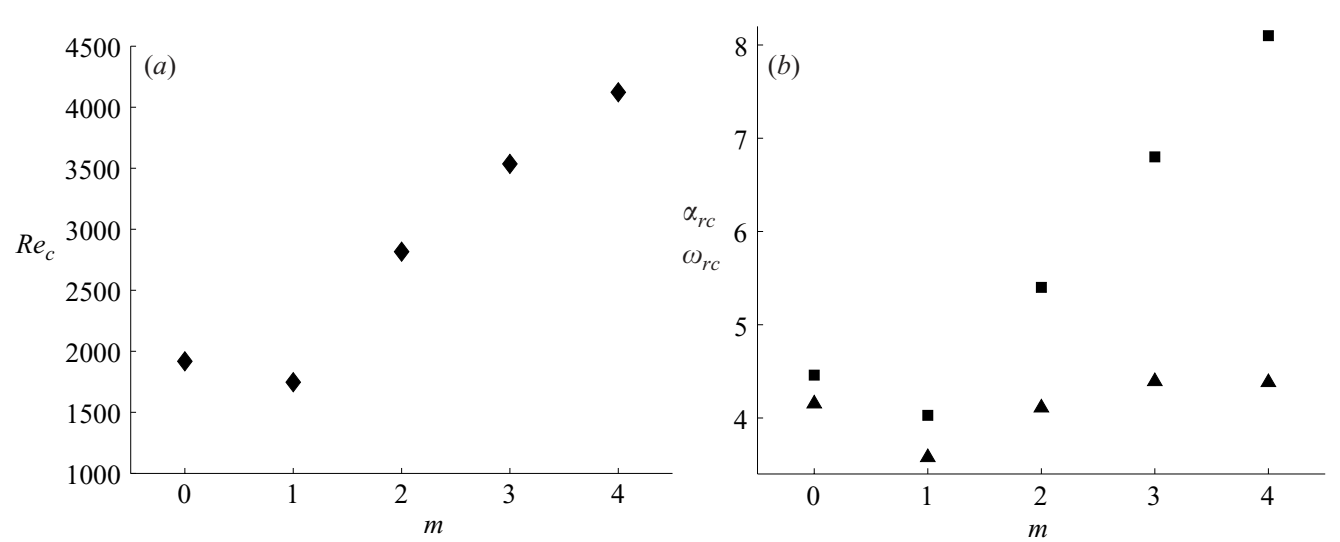

FiguRE 15. (a) Critical Reynolds number and $(b)$ critical frequency $(\triangle)$ and streamwise wavenumbers $(\square)$, for optimally perturbed base flows with $\epsilon=10^{-5}$, as a function of the azimuthal wavenumber $m$.

points in this figure follow a relation of the kind $\epsilon=R e_{c}^{\gamma}$ with $\gamma=-2$ or, equivalently, in terms of a velocity amplitude: $\epsilon_{a}=\sqrt{\epsilon}=R e_{c}^{\gamma_{a}}$ with $\gamma_{a}=-1$. When the same kind of analysis is performed for plane Couette flow (see Bottaro et al. 2003), an identical value of the exponent is obtained. Moreover, a similar behaviour is found by a number of authors for plane Poiseuille and plane Couette flows (see, for example, Trefethen et al. 1993) for the case where $\epsilon_{a}$ represents the norm of a generic disturbance operator to the Orr-Sommerfeld operator. The value of their exponent, however, turns out to be $\gamma_{a}=-2$, i.e. twice as big as ours. This discrepancy is ascribed to the fact that the disturbance operator in our analysis is configured in a very specific form, i.e. as a perturbation of the base-flow velocity only (see Bottaro et al. 2003), thus leading to a more conservative value of the minimal norm of the destabilizing perturbation. We further note that Trefethen et al. (2000) have assembled a number of experimental, theoretical and numerical studies based on which they deduce a value of $\gamma_{a}$ for pipe flow equal to $-3 / 2 \pm 0.3$. The value of the exponent found here is reasonably close to their estimate. The behaviour of the critical wavenumber with $\epsilon$ also follows a power law, evidenced in figure 14(b), and so does the critical frequency (not shown.)

Finally, in figure 15(a) we have plotted the critical Reynolds number as a function of the azimuthal wavenumber $m$ for a norm of the deviation equal to $\epsilon=10^{-5}$. The corresponding values of the critical frequency and streamwise wavenumber are shown in figure $15(b)$. The $m=1$ mode appears to be the most prone to destabilization by the mechanism discussed here. The structure of this critical mode for $m=1$ is displayed in figure 16 by means of a plot of $(v, w)$ velocity vectors in the $(r, \theta)$-plane together with isolines of the axial velocity $u$. We observe that intense disturbances are concentrated in the proximity of the cylinder axis. For increasing values of $R e$, the perturbations are found to shift towards the wall, as already discussed in the context of figure $12(b)$. In figure 17, the axisymmetric critical mode is drawn over one wavelength in the axial direction. This is a meridional mode (cf. Tumin 1996), with the azimuthal velocity component $w$ identically zero. This axisymmetric mode is the second most unstable mode (cf. figure 15(a)) and it can play a role in the transition process when $R e$ exceeds 2000. 

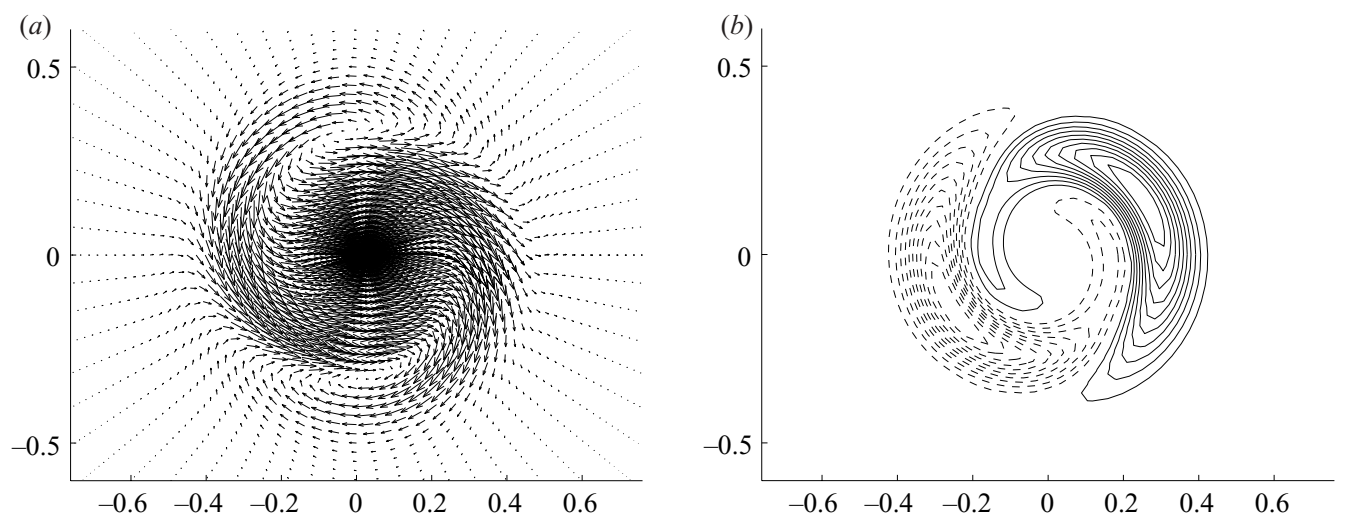

Figure 16. $(a)$ Vector representation of $(v, w)$ velocity components in the $(r, \theta)$-plane and (b) contours of $u$-component (dashed lines indicate negative values), for the neutral mode at $R e=1746.6, m=1, \omega=3.5775, \epsilon=10^{-5}$.

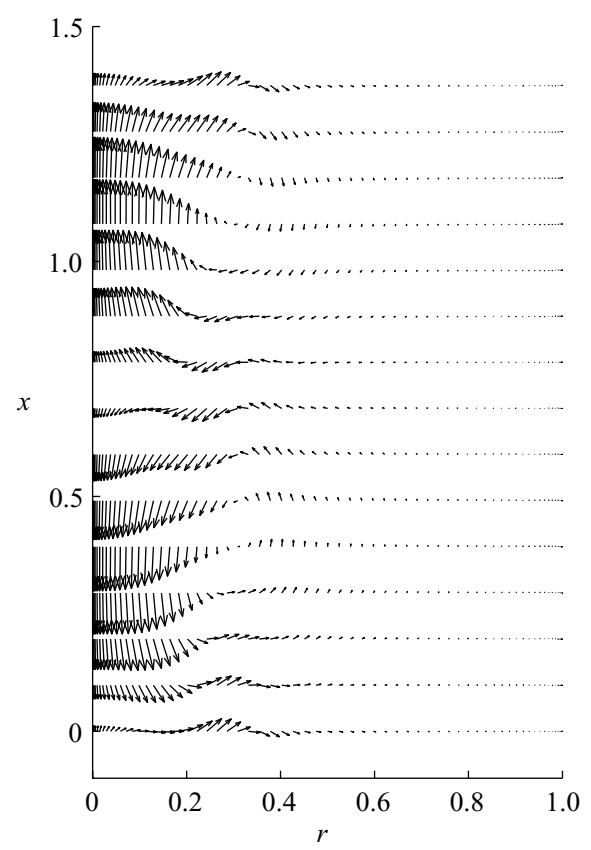

FIGURE 17. Representation in the $(r, x)$-plane of the velocity vectors for the neutral mode at $R e=1918, m=0, \omega=4.1529, \epsilon=10^{-5}$.

\section{Numerical simulations}

We now study the transition scenario related to 'optimal base-flow distortions', by means of direct numerical simulations: these will demonstrate how transition proceeds after the initial stage and allow a detailed study of some transition scenarios. For the simulations we use a code developed by Eggels et al. (1994). This code computes the flow in a cylindrical pipe geometry with a finite-volume technique with second-order accuracy for the spatial discretization. The time advancement uses a leap-frog scheme, 
with second-order accuracy for the discretization of the advective terms and a forward Euler scheme over two time steps for the diffusive terms. For further details, refer to Eggels et al. (1994). The code has been extensively checked by reproducing the results obtained by Ma et al. (1999), who have used a spectral code. The agreement is found to be excellent and this gives us the necessary confidence that the transition process can be adequately simulated with the present second-order finite-volume code.

After these tests, the code has been modified to simulate the spatial development of the disturbance velocity $\boldsymbol{v}=(u, v, w)$ evolving on top of a given axisymmetric, not necessarily parabolic, base flow velocity distribution $U(r)$. The resulting set of equations reads:

$$
\begin{gathered}
\frac{\partial u}{\partial x}+\frac{1}{r} \frac{\partial(r v)}{\partial r}+\frac{1}{r} \frac{\partial w}{\partial \theta}=0 \\
\frac{\partial u}{\partial t}+(U+u) \frac{\partial u}{\partial x}+v \frac{\partial u}{\partial r}+v \frac{\mathrm{d} U}{\mathrm{~d} r}+w \frac{1}{r} \frac{\partial u}{\partial \theta}=-\frac{\partial p}{\partial x}+\frac{1}{R e} \nabla^{2} u \\
\frac{\partial v}{\partial t}+(U+u) \frac{\partial v}{\partial x}+v \frac{\partial v}{\partial r}+w \frac{1}{r} \frac{\partial v}{\partial \theta}=-\frac{\partial p}{\partial r}+\frac{1}{R e}\left(\nabla^{2} v-\frac{v}{r^{2}}-\frac{2}{r^{2}} \frac{\partial w}{\partial \theta}\right) \\
\frac{\partial w}{\partial t}+(U+u) \frac{\partial w}{\partial x}+v \frac{\partial w}{\partial r}+w \frac{1}{r} \frac{\partial w}{\partial \theta}=-\frac{1}{r} \frac{\partial p}{\partial \theta}+\frac{1}{R e}\left(\nabla^{2} w-\frac{w}{r^{2}}+\frac{2}{r^{2}} \frac{\partial v}{\partial \theta}\right)
\end{gathered}
$$

where $\nabla^{2}$ is again given by $(2.3)$.

In these equations, no additional forcing term is needed to produce and maintain the base flow $U(r)$. At the end of the computational domain, a fringe-region technique is implemented, following closely the method proposed by Lundbladh et al. (1994), who apply this method in their spectral code to combine the spatial evolution of the flow with periodic boundary conditions in the streamwise direction. In our case, the fringe region is used to gradually damp the disturbances flowing out of the physical domain with minimal reflection. This proves to be necessary to eliminate numerical wiggles originating at the outflow boundary. At the inflow boundary, the unstable mode of the optimally perturbed base flow $U(r)$ is imposed with a prescribed amplitude.

The results of the simulations are analysed in terms of the kinetic energy of the disturbance velocity split up in its individual Fourier components according to:

$$
E_{d i s}^{(m, n)}(x)=\sum_{j= \pm m} \sum_{k= \pm n} \frac{1}{T} \int_{\tau}^{\tau+T} \int_{0}^{2 \pi} \int_{0}^{1} \frac{1}{2}\left|\widehat{\boldsymbol{v}}(r, x ; j, k) \mathrm{e}^{\mathrm{i}(j \theta-k \omega t)}\right|^{2} r \mathrm{~d} r \mathrm{~d} \theta \mathrm{d} t .
$$

Here, $T=2 \pi / \omega$ and $\tau$ is an arbitrary starting time, subject to the condition that the asymptotic temporal state has been reached. The number pair $(m, n)$ indicates the harmonics, where $m$ is the wavenumber in the angular direction and $n$ the multiple of the non-dimensional frequency $\omega$. At the inflow, a disturbance is prescribed with a given value of $(m, n)$, which is called the fundamental mode. During the transition process, wavenumbers will be excited by nonlinear triad interactions that are multiples of the fundamental mode and these are called higher harmonics. Equation (4.2) shows that the spectral energy for each $(m, n)$ is computed as the sum over the modes $( \pm m, \pm n)$.

\subsection{Mode $m= \pm 1$ and $n= \pm 1$ as inflow condition}

We consider, in this case, the unstable mode illustrated in figure 8, which refers to the optimally modified base flow shown in figure 5 . The parameters for this flow are 


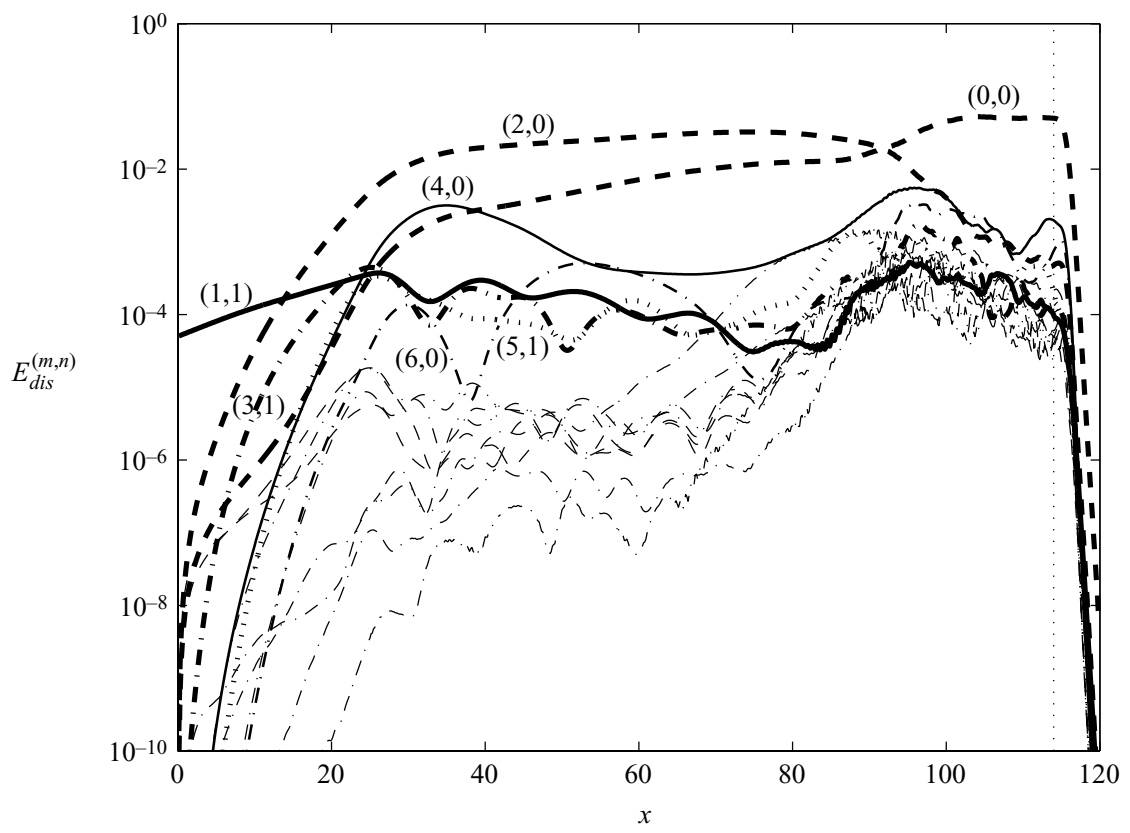

FIGURE 18. Spatial evolution of the disturbance kinetic energy for the various Fourier modes $(m, n)$, with $\omega=0.5$ fundamental frequency. The inflow condition consists of a combination of the unstable eigenmodes with $m= \pm 1, n= \pm 1$ at $R e=3000$ and for $\epsilon=2.5 \times 10^{-5}$, each one with amplitude $A_{v}=0.002$. The dotted vertical line indicates the start of the fringe region.

$m=1, R e=3000, \omega=0.5$ and $\epsilon=2.5 \times 10^{-5}$. The computational domain that we have used for this simulation measures $120 R$ in the streamwise direction. By extensive tests, it is found that an acceptable resolution is achieved by employing $64 \times 32 \times 768$ grid points in the radial, azimuthal and axial directions, respectively, on a uniform grid in all directions. The inflow condition is a linear combination of the unstable eigenmodes with $(m, n)=( \pm 1, \pm 1)$ in order to generate a real-valued velocity field, as required by the DNS code. The flow field at $x=0$ consists, therefore, of a standing azimuthal wave. The amplitude of this wave is set equal to $A_{v}=0.002$, so that the disturbance kinetic energy at $x=0$ amounts to $E_{d i s}(0) \simeq 5 \times 10^{-5}$. This initial amplitude has been chosen sufficiently small in order to observe the exponential growth of the disturbance in the first part of the pipe, but high enough to excite transition in the downstream portion of the computational domain.

The results of the simulation in terms of kinetic energy of the individual Fourier components $(m, n)$ are presented in figure 18. We note that in this case, for which the initial disturbance is given by $(m, n)=( \pm 1, \pm 1)$, only modes with $(m+n)$ even can be generated by quadratic interactions.

At the inflow, only the $(1,1)$ mode is present. Its energy as a function of $x$ shows the initial exponential growth at the rate dictated by linear theory. This growth would not have been observed if the parabolic Hagen-Poiseuille flow had been used as the base flow. In that case, the $(1,1)$ mode would have started to decay immediately as, for instance, found by Ma et al. (1999). Subsequently, the harmonic modes $(0,0)$ and $(2,0)$ generated by the quadratic self-interaction of the fundamental modes and indicated in the figure by dashed lines, start to grow (to a lesser extent also $(0,2)$ and $(2,2)$ show some amplification, but this seems less important). Mode $(4,0)$ (thin 


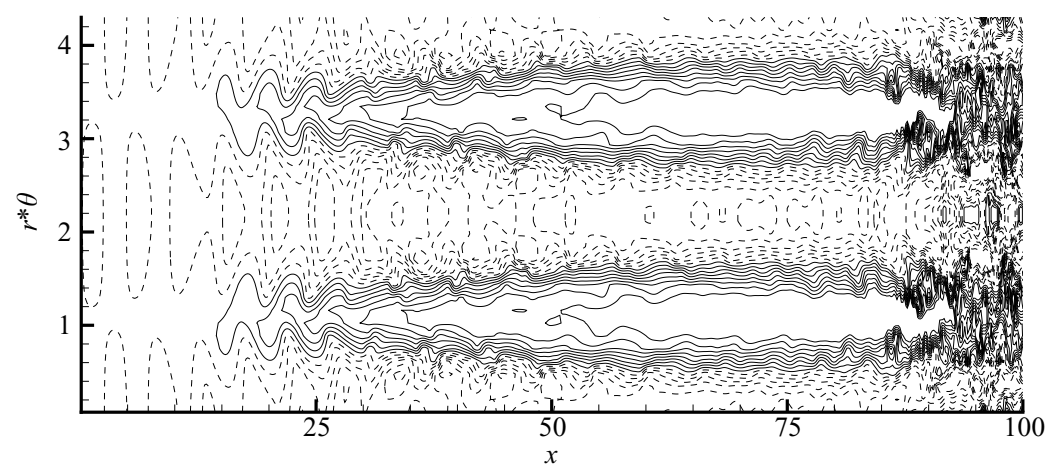

FIGURE 19. Instantaneous streamwise disturbance velocity at $r=0.7$ : contour lines range from -0.17 to 0.2668 , spaced by 0.0336 .

solid line) appears also to grow rapidly. The very rapid growth of the steady $(2,0)$ and $(4,0)$ modes up to $x \simeq 30$ can be associated with transient growth. In the first part of the pipe, mode $(2,0)$ has the form of streamwise vortices centred at the radial position corresponding to the inflection point $s_{1}$ in the distorted mean profile (see figure 5), i.e. quite close to the pipe wall. This allows for an effective lift-up of low-speed fluid from the wall towards the centre of the pipe. This results further downstream in the formation of a strong streaky structure, which is illustrated in figure 19. The axial component of the velocity perturbation is here two orders of magnitude larger than the other two velocity components.

Figure 19 also shows that around $x \simeq 25$, a secondary subharmonic odd instability appears. It takes the form of varicose oscillations in the low-speed streaks (drawn with dashed lines) and sinuous oscillations in the high-speed streaks (solid lines) (see figure 2 of Andersson et al. (2001) for further details of these subharmonic secondary modes).

We have seen in the previous section that, for a given value of $\epsilon$, the optimal baseflow distortion moves towards the centreline for decreasing values of the Reynolds number (see figure 12(b)). This would weaken the lift-up effect and, correspondingly, the flow becomes more stable (to transient amplification).

The conclusion for the initial stage behaviour for this $(m= \pm 1, n= \pm 1)$ case must be that, despite the initial exponential growth, transient growth still plays an important role in the transition process. This has already been indicated by Bottaro et al. (2003) who, in a similar analysis for plane Couette flow, showed that the linear stability operator of the perturbed base flow remains highly non-normal. It can be argued that in this transition scenario there is combination rather than a competition between the two growing mechanisms, modal and non-modal, the second being enhanced by the first.

After this initial phase, in which linear growth mechanisms and quadratic selfinteractions of the fundamental modes dominate, the growth of the $(2,0)$ mode is damped mostly by strong nonlinearities producing a mean flow modification, i.e. $(2,0)+(-2,0) \rightarrow(0,0)$. Furthermore, the coupling between different disturbance components plays an important role in redistributing the energy in wavenumber space. In particular, it is clear from figure 18 that low-frequency modes (for example modes $(3,1),(4,0),(5,1)$ and $(6,0))$ dominate over other nonlinearly generated modes with higher wavenumbers, which do not possess the potential to grow by linear mechanisms. This was already observed by Ma et al. (1999) and Henningson, 
Lundbladh \& Johansson (1993), who speak, respectively, of an $m$-cascade in HagenPoiseuille flow or a $\beta$-cascade in plane Poiseuille flow, in which the disturbance energy is mainly transferred through a route with increasing azimuthal or spanwise wavenumber.

Beyond the downstream position where the energy of the $(2,0)$ mode reaches a peak, i.e. at $x \simeq 75$, a second phase of rapid growth of higher-order modes, i.e. modes with higher harmonics of $\omega$, is responsible for the final breakdown to turbulence.

A sequence of events comparable with the case described above was also found by Berlin et al. (1994) for a boundary-layer flow, in which transition was initiated by a pair of oblique waves, which could grow transiently in the initial stage and whose quadratic interaction resulted in the generation of streamwise vortices. These vortices, in turn, produced streaks by the lift-up mechanism, eventually bringing the flow to transition via a secondary instability of the streaky flow. Our case, however, differs from that of Berlin et al. (1994), because our process starts with the primary instability of the modified base flow, i.e. the initial energy in the streamwise vortices is fed by the exponential growth of the unstable mode.

In a simulation with the amplitude of each unstable mode at the inflow equal to $A_{v}=0.001$, i.e. for $E_{d i s}(0) \simeq 1.25 \times 10^{-5}$, the second phase of rapid growth of the higher-order frequency harmonics and the ensuing transition to turbulence did not occur. Instead, a decay of the streaks after the peak in the energy of the $(2,0)$ mode was observed. Therefore, in this scenario starting with the exponential growth of small disturbances on a modified base flow, which can directly generate streamwise vortices and streaks, the essential feature, which determines whether transition occurs or not, is the final amplitude of the streaks. This amplitude must reach a threshold value in order to force the final breakdown. It can thus be stated that the whole transition process in this case is dominated by the evolution and breakdown of the large-amplitude streaks, as shown in figure 19. Therefore, this scenario could go under the classification of oblique transition (see Schmid \& Henningson 2001), the only difference being that here the infinitesimal oblique (in our case actually helical) waves can initially grow exponentially. The subsequent series of events becomes then qualitatively similar to that observed in numerical simulations initiated by the nonmodal amplification of optimal initial vortices (see O'Sullivan \& Breuer 1994b; Ma et al. 1999).

\subsection{Mode $m=0$ and $n= \pm 2$ as inflow condition}

Our analysis of optimal base-flow deviations has also shown that axisymmetric disturbances can experience exponential amplification. Let us consider this case with a superposition of modes $(0, \pm 2)$ as initial condition. For such a disturbance, quadratic self-interaction cannot produce modes such as $(2,0)$, which, as we have seen in the previous section, play a central role in the oblique transition process. Therefore, we expect, in this case, a different transition scenario and its elucidation is the objective of our second numerical simulation.

The computational domain used in this second case measures $80 R$ in the streamwise direction, with a resolution of $64 \times 32 \times 640$ grid points in the radial, azimuthal and axial directions, respectively. We use the optimally modified base flow at $R e=3000$, and $\epsilon=2.5 \times 10^{-5}$, which destabilizes an axisymmetric disturbance with frequency $\omega=1$ (note that $\omega=0.5$ is still used as the fundamental frequency in the Fourier decomposition). Stability theory predicts a growth rate of the unstable mode equal to $-\alpha_{i}=0.07955$. The inflow condition consists of a combination of the $(0, \pm 2)$ Fourier modes to obtain a real inflow velocity field and the amplitude was chosen equal to 


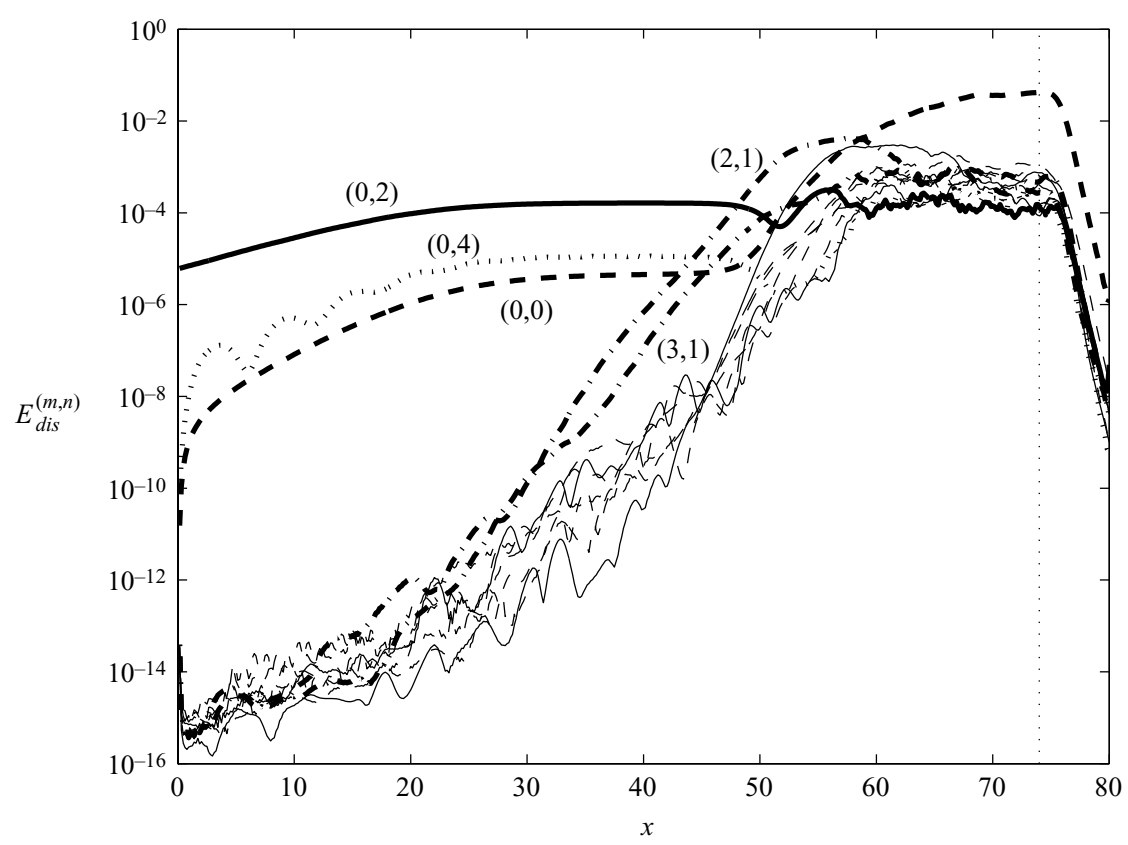

Figure 20. Spatial evolution of the disturbance kinetic energy for various Fourier modes $(m, n)$, with $\omega=0.5$ fundamental frequency. The inflow condition consists of a combination of the unstable eigenmodes at $R e=3000, m=0, n= \pm 2, \epsilon=2.5 \times 10^{-5}$, each one with $A_{v}=0.001$, plus small-amplitude random perturbations. The dotted vertical line indicates the start of the fringe region.

$A_{v}=0.001$, i.e. $E_{d i s}(0) \simeq 6.3 \times 10^{-6}$. It was found that, in order to have transition for this case, small random perturbations had to be added to the inflow velocity field. The magnitude of these random perturbations was set equal to $1 \%$ of the magnitude of the primary disturbance.

The results of the numerical simulation in terms of $E_{d i s}^{(m, n)}(x)$ are shown in figure 20. Initially, the exponential growth of mode $(0,2)$ is observed in agreement with theoretical predictions. Almost immediately, modes $(0,0)$ and $(0,4)$ are produced by quadratic interactions. Eventually, mode $(0,2)$ saturates and, around $x \simeq 40$, a secondary instability appears, introducing the subharmonic mode $(2,1)$. In this phase mode $(3,1)$ also becomes important. These modes, which allow for an azimuthal modulation of the flow, lead to the late stages of transition and to the final breakdown to turbulence towards $x \simeq 60$.

In figure 21, we show a contour plot of the streamwise component of the disturbance velocity at $r=0.7$. The initial axisymmetric nature of the disturbance is clear in this figure. The subharmonic instability can be identified as a transverse wave in the initially axisymmetric disturbance pattern, leading to a staggered array of $\Lambda$-vortices. Such a transition scenario is strongly reminescent of the $H$-type transition initiated by two-dimensional Tollmien-Schlichting waves in a flat-plate boundary layer.

\subsection{Relaxation of the optimal base-flow distortion}

Both the variational analysis and the numerical simulations discussed in the previous sections are based on the assumption that the optimally disturbed base flow is 


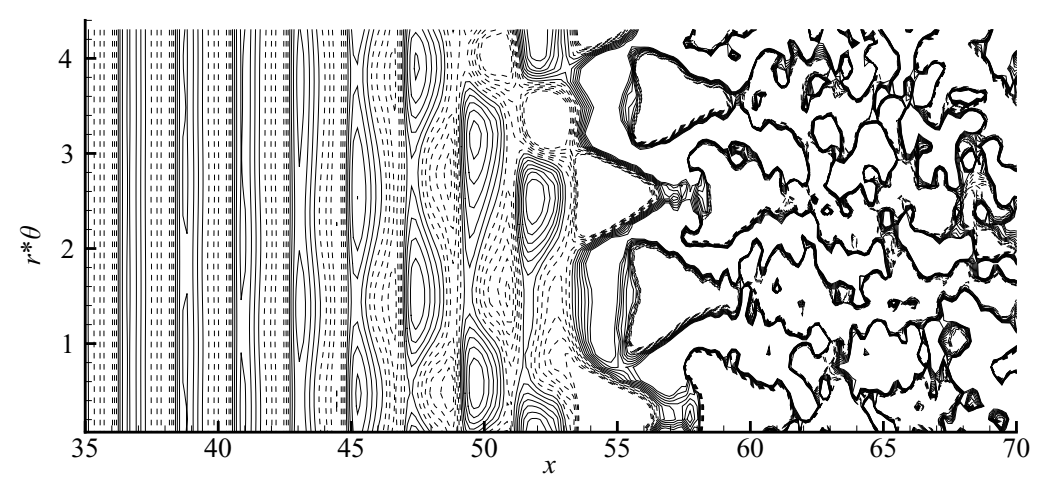

FIGURE 21. Instantaneous streamwise disturbance velocity at $r=0.7$ : contour lines range from -0.03 to 0.03 , spaced by 0.005 .

established and that it persists over the whole length of the domain. Given this assumption, the numerical simulations have shown that a slightly modified base flow can become linearly unstable and that, if the initial disturbance amplitude is large enough, nonlinear processes can complete the transition to turbulence.

We now consider the case in which the base-flow distortion is present only over a finite length of the pipe and it relaxes downstream because of, for instance, viscous diffusion. The question is: does the instability mode have enough time to grow and induce nonlinear interactions, or will it be damped because of the relaxation of the base flow to the ideal (linearly stable) profile?

To answer this question for the case that we have considered in $\S 4.1$, we have carried out a numerical simulation, in which the optimal deviation from the parabolic profile is imposed only at the inflow section and it is then allowed to diffuse downstream. This implies that the Hagen-Poiseuille profile is used as the base flow in (4.1) throughout the rest of the domain. In this simulation, the norm of the base-flow deviation is $2.5 \times 10^{-5}$, larger than the critical distortion value at $R e=3000$, which is around $3 \times 10^{-6}$ (cf. figure 14(a)). Because of this relatively small initial distortion, the exponential growth of the disturbance will be slow. In a real situation, however, the base-flow distortion will probably be present along a finite length of the pipe, and not only at a single cross-section as considered here. As a result of the amplification over this region of perturbed base flow, a higher value of the unstable disturbance energy will result at the end of the region.

The computational domain, the resolution and the inflow perturbation are the same as those used in the simulation reported in $\S 4.1$. If the same amplitude of the inflow disturbance is used, i.e. $A_{v}=0.002$, no transition is observed and mode $(1,1)$ is only slightly amplified initially, and it decays afterwards. The amplitude $A_{v}$ has to be increased up to 0.0065 in order to observe transition, which, as follows from figure 22, occurs upstream of the transition point found in $\S 4.1$. The growth of the $(1,1)$ mode is exponential only in the first downstream positions and later it is sustained by nonlinear interactions with higher harmonics. The $(0,0)$ mode starts from a finite value at $x=0$, which represents the imposed base-flow deviation. Notice again that this is very small; indeed, it is smaller than the inflow energy of mode $( \pm 1, \pm 1)$.

In figure 23 , a more detailed view of the energy of modes $(1,1),(2,0)$ and $(0,0)$ is shown for the two initial amplitude values, i.e. $A_{v}=0.002$ (dashed lines) and 


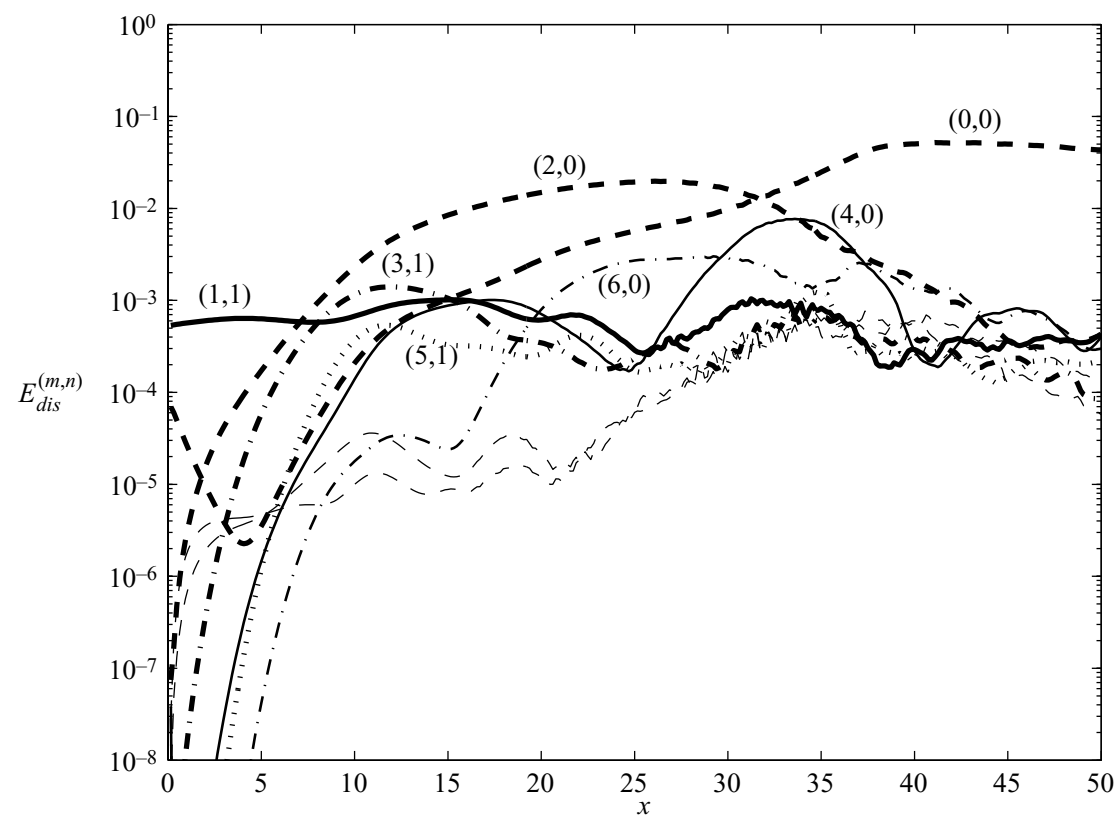

FIGURE 22. Spatial evolution of the disturbance energy for the various Fourier modes $(m, n)$, with $\omega=0.5$ fundamental frequency. The initial amplitude of each mode $( \pm 1, \pm 1)$ is $A_{v}=0.0065$.

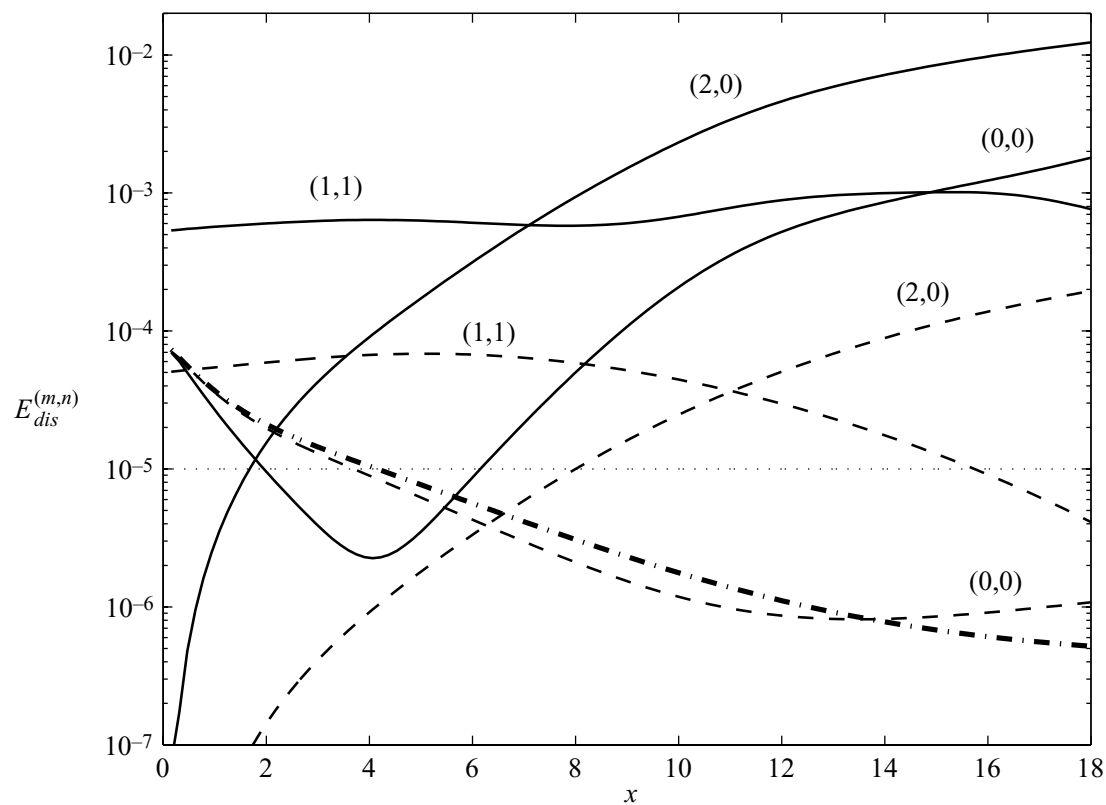

FIGURE 23. Initial evolution of the disturbance energy for the various Fourier modes $(m, n)$, with $\omega=0.5$ fundamental frequency. The solid lines refer to an initial amplitude of each mode $( \pm 1, \pm 1)$ equal to $A_{v}=0.0065$, the dashed lines to $A_{v}=0.002$. The thick dash-dotted line represents the spatial evolution of the mean flow deviation only, owing to viscous diffusion. 
$A_{v}=0.0065$ (solid lines). In the figure we also show the evolution of the energy of the mean flow distortion alone (thick dash-dotted line), and its threshold value at $R e=3000$ (dotted horizontal line), i.e. the value below which the exponential growth of the $(1,1)$ mode is no longer sustained. The mean flow distortion is observed to decay rapidly at this relatively low value of the Reynolds number. When its energy goes below the threshold, the $(2,0)$ mode for the case with the higher value of $A_{v}$ has an energy comparable to that of mode $(1,1)$. Therefore, the streamwise vortices and the resulting streaks are strong enough to influence, by means of nonlinear interactions with the $(3,1)$ mode (not shown), the evolution of the $(1,1)$ mode, which thus can grow further. For the lower value of $A_{v}$, when the threshold is crossed at $x$ close to 4 , the $(2,0)$ mode is still very weak so that the growth of the $(1,1)$ mode cannot be sustained either by linear or by nonlinear mechanisms and consequently the initial perturbation decays. Therefore, also in this case we can conclude that the occurrence of transition is influenced by the amplitude of the streaks downstream of the position where the distortion has been introduced.

A similar numerical simulation has also been performed for the axisymmetric disturbance analysed in $\S 4.2$. When the base-flow perturbation is applied only at the inflow, we find that, owing to the rapid diffusion of the optimal base-flow distortion, the exponential growth of the axisymmetric unstable mode is confined to a distance of only a few pipe diameters. From that point on, any further growth of the initial perturbation mode $(0,2)$ and of the nonlinearly generated modes $(0,0)$ and $(0,4)$ is excluded, since non-modal effects are very weak for axisymmetric disturbances (see Schmid \& Henningson 2001). Even an increase in the value of the initial disturbance amplitude up to $A_{v}=0.01$ was not sufficient to provoke transition in the flow.

To find the reason for this failure to produce transition we consider the total energy of the disturbance calculated as the sum over all modes $m$ and $n$, which reads:

$$
E_{d i s}(x)=\sum_{m} \sum_{n} E_{d i s}^{(m, n)}(x) .
$$

The behaviour of $E_{d i s}$ as a function of $x$ for the cases considered in $\S \S 4.1$ and 4.2, where the base-flow distortion was imposed over the whole length of the pipe, is presented in figure 24 , in which we also show the exponential growth of $E_{d i s}$ as a result of linear theory.

For the case with the $( \pm 1, \pm 1)$ inflow condition, the nonlinearity is clearly destabilizing, resulting in a faster than exponential growth of the energy, implying that transition occurs via a subcritical bifurcation from the base state (Barkley \& Tuckerman 1999). On the other hand, for the $(0, \pm 2)$ inflow condition, the nonlinearity clearly saturates the linear instability resulting in a lower than exponential growth of the energy and in the supercritical nature of the bifurcation.

Therefore, we conclude that for the latter scenario to be observed, the optimal base-flow deviation must persist over a finite length of the pipe and the induced growth (i.e. the norm of the deviation itself) must be strong enough for the late stage to set in before the base-flow deviation is smeared out by viscosity. This is confirmed by two additional simulations, in which the optimal base-flow distortion is forced along a finite length of the pipe extending from the inflow section up to $x=40$ and $x=50$, respectively, and it is then allowed to relax further downstream. For these simulations, the Navier-Stokes equations are solved for the complete velocity field 

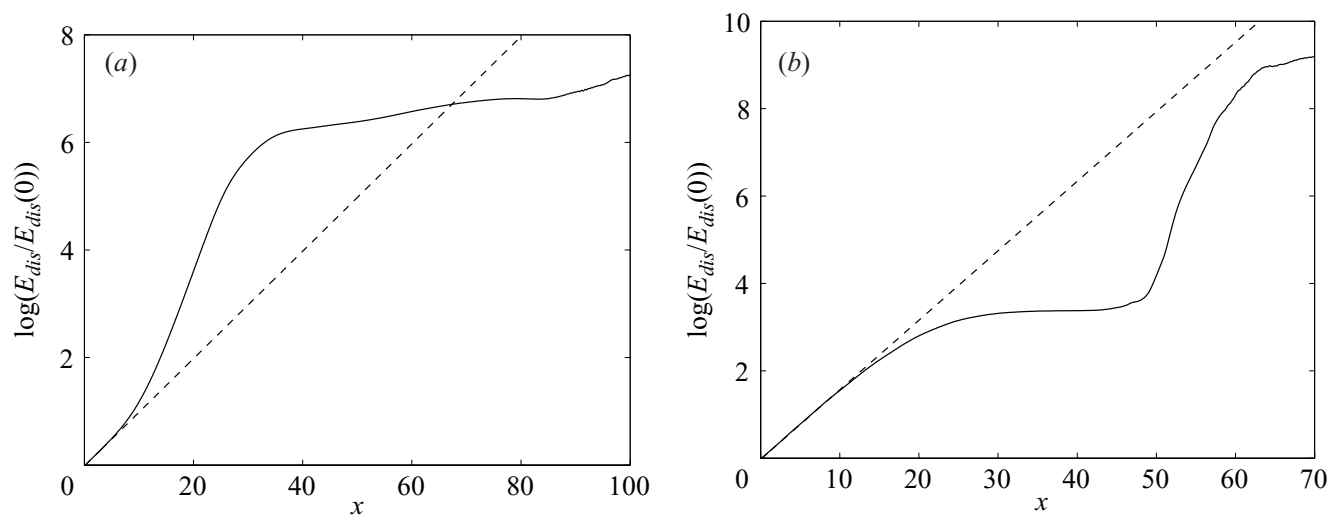

FIGURE 24. Spatial evolution of the total disturbance energy, for the case in which the optimal base-flow distortion is maintained over the whole length of the pipe. $(a)$ The inflow condition is $( \pm 1, \pm 1)$. (b) The inflow condition is $(0, \pm 2)$. The dashed lines indicate the exponential growth predicted by linear theory for the two cases.
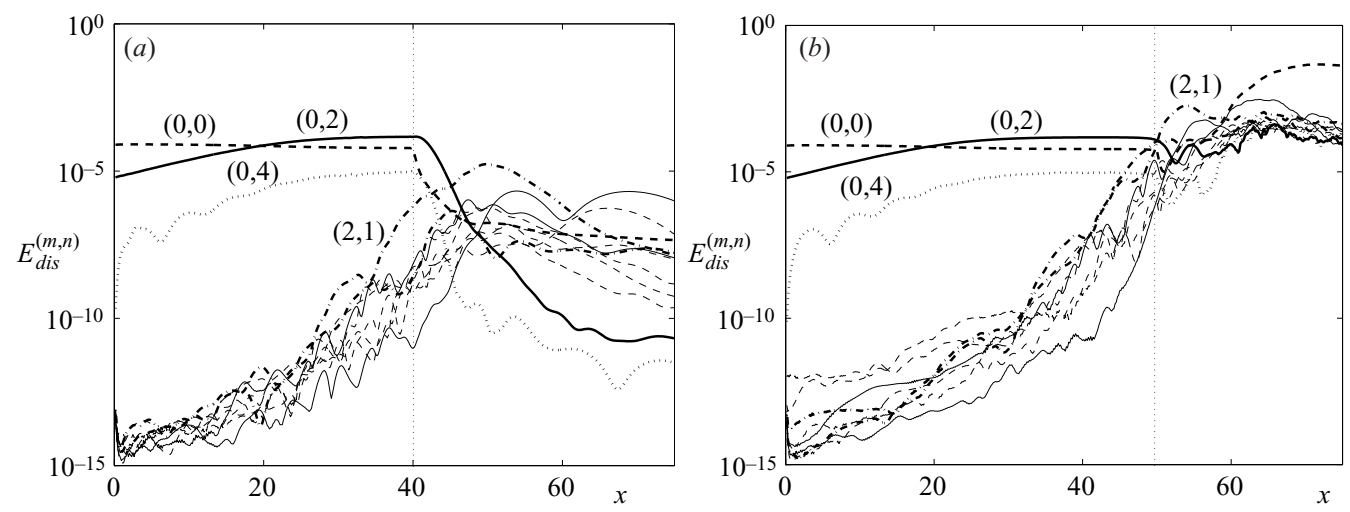

FiguRE 25. Spatial evolution of the disturbance energy for the various Fourier modes $(m, n)$, with fundamental frequency $\omega=0.5$. The optimal base-flow distortion is forced up to $x=40$ in (a) and up to $x=50$ in $(b)$. The initial amplitude of each mode $(0, \pm 2)$ is equal to $A_{v}=0.001$ in both cases.

and not only for the disturbance velocity, as it was done in (4.1). The base-flow distortion is imposed along the prescribed length $L$ of the pipe by adding locally a forcing term in the $u$-momentum equation of the kind $f_{x}=-\nabla^{2}(\Delta U) / R e$. All the other parameters are left unchanged with respect to those described in $\S 4.2$. The results are presented in figures $25(a)$ and $25(b)$, for $L=40$ and $L=50$, respectively. Transition is observed only when $L=50$, because, in this case, the secondary instability mode $(2,1)$ has grown, at $x=L$, to a sufficient level for transition to proceed. Clearly, a higher level of random disturbances at the inflow would have resulted in an earlier development of the secondary instability and would also have led to transition under the conditions of figure 25(a). Moreover, in the case where the optimal baseflow distortion is imposed up to $x=40$, transition has also been observed after 


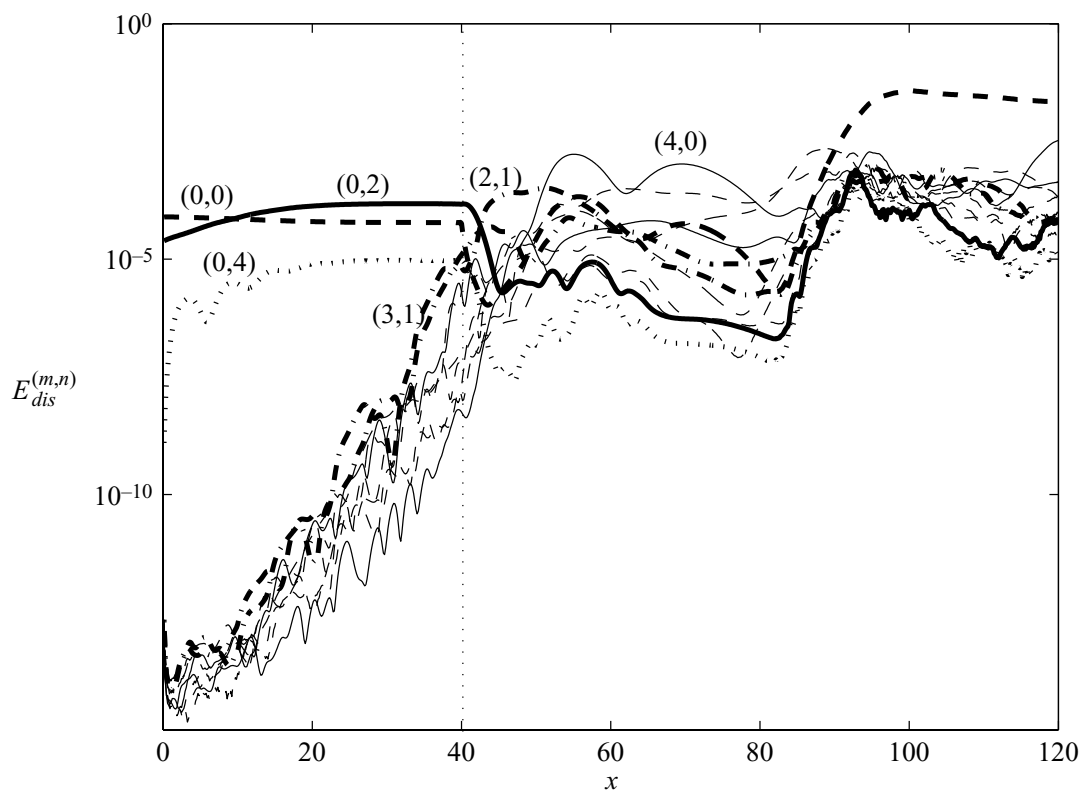

FIGURE 26. Spatial evolution of the disturbance energy for the various Fourier modes $(m, n)$, with fundamental frequency $\omega=0.5$. The optimal base-flow distortion is forced up to $x=40$ and the initial amplitude of each mode $(0, \pm 2)$ is $A_{v}=0.002$.

increasing the amplitude of the inflow disturbance by a factor of two, as shown in figure 26.

Therefore, the higher the level of the periodic inflow disturbance and/or random noise, the higher the chance of observing the second transition scenario. This chance is also increased when higher values of the Reynolds number and/or higher values of the amplitude of the distortion are considered. We hypothesize that these were the conditions that probably led to the observation of this type of transition scenario in the early experiments by Leite (1959), in which axisymmetric disturbances were generated either by oscillating a thin sleeve adjacent to the inner wall of the pipe or by a ring airfoil mounted within the pipe at a radial position $r \simeq 0.72$ (i.e. a very receptive position at reasonably large values of the Reynolds number). Small disturbances introduced by the periodic motion of the sliding sleeve decayed as they travelled downstream for all the Reynolds numbers investigated, thus confirming the theoretical results on the linear stability of Hagen-Poiseuille flow to axisymmetric disturbances. However, it was mentioned that 'upon introducing the ring airfoil disturbance amplitudes were increased appreciably and the stability characteristics of the flow changed markedly'. More precisely, at $R e=12000$, transition was observed even when the airfoil was held stationary, whereas at $R e=8000$, transition occurred when the airfoil oscillated with the smallest possible amplitude. These results suggest (as was already noticed by Gill 1965) that the change in the mean velocity profile induced by the wake downstream of the ring airfoil was sufficient to cause transition to turbulence at high Reynolds numbers. 


\subsection{Discussion}

Bottin et al. (1998) have given experimental evidence for the effect that small baseflow distortions have on the stability characteristics of subcritical shear flows. They found that finite-amplitude solutions, in the form of streamwise vortices, bifurcate subcritically from a plane Couette flow slightly modified (and destabilized) by a thin spanwise wire in the zero-velocity plane (i.e. at the position that returns the greatest effects in the destabilization of the mean flow, see Gill 1965; Bottaro et al. 2003). They also found that this streamwise-streak state still bifurcates when the diameter of the wire is gradually decreased to zero, i.e. when the distortion of the mean speed vanishes. Moreover, the streamwise extent of the streaks is much larger than the region where the profile is significantly modified by the presence of the wire, suggesting that the flow has switched to a new state that has little to do with the modified basic state as results also from our calculations. These results have been confirmed by numerical simulations performed by Barkley \& Tuckerman (1999), who also indicated that small geometric perturbations destabilize plane Couette flow via a subcritical instability and that the bifurcating solution is a three-dimensional flow with streamwise vortices.

This method of looking for non-trivial solutions to the fully nonlinear problem, i.e. for equilibrium states different from the basic one, by considering a modified system with known solutions and then progressively deforming it to recover the original problem, is known as the continuous deformation approach (Bottin et al. 1998). It has already been used in the past to identify finite-amplitude solutions in plane Couette flow by a number of authors (see, for instance, Nagata 1990; Clever \& Busse 1992) and suggests a possible application of the present investigation for the identification of coherent states in pipe flow. Their existence, in the form of travelling waves, has been recently confirmed numerically by Faisst \& Eckhardt (2003) with the help of a continuation method. At low Reynolds numbers, twodimensional downstream elongated vortices are added to the base flow, by means of a volume force that is increased up to the first bifurcation leading to a fully threedimensional travelling-wave solution (this corresponds in our case to the forcing of the mean flow distortion, bringing about streamwise vortices and streaks). Tracking this state in parameter space, by decreasing the body force and increasing the Reynolds number, Faisst \& Eckhardt (2003) could finally reach a finite-amplitude three-dimensional travelling-wave state for a vanishing body force. In this way the authors identified several families of solutions with different symmetries. By looking at the flow fields resulting from our simulations, we could identify several structures resembling the solutions computed by Faisst \& Eckhardt (2003). For example, from the results of the simulation reported in $\S 4.2$, shown in figures 20 and 21 , we could identify at $x \simeq 56$, a structure that seems closely related to the two-fold state of figure 3 in Faisst \& Eckhardt (2003). As can be seen from figure 27, it consists of two pairs of counter-rotating vortices that generate four streaks of fast fluid close to the wall and two streaks of slow fluid towards the centre. The modes that mostly contribute to this three-dimensional structure are $(2,1),(0,4)$ and $(0,0)$, as can be deduced from their energy levels in figure 20 at $x \simeq 56$. Notably, this state can also be observed, at about the same downstream position, in the simulation for which the optimal base-flow distortion is imposed up to $x \simeq 50$ ( $\mathrm{cf}$. the results given in figure 25 where the same modes are most relevant). 


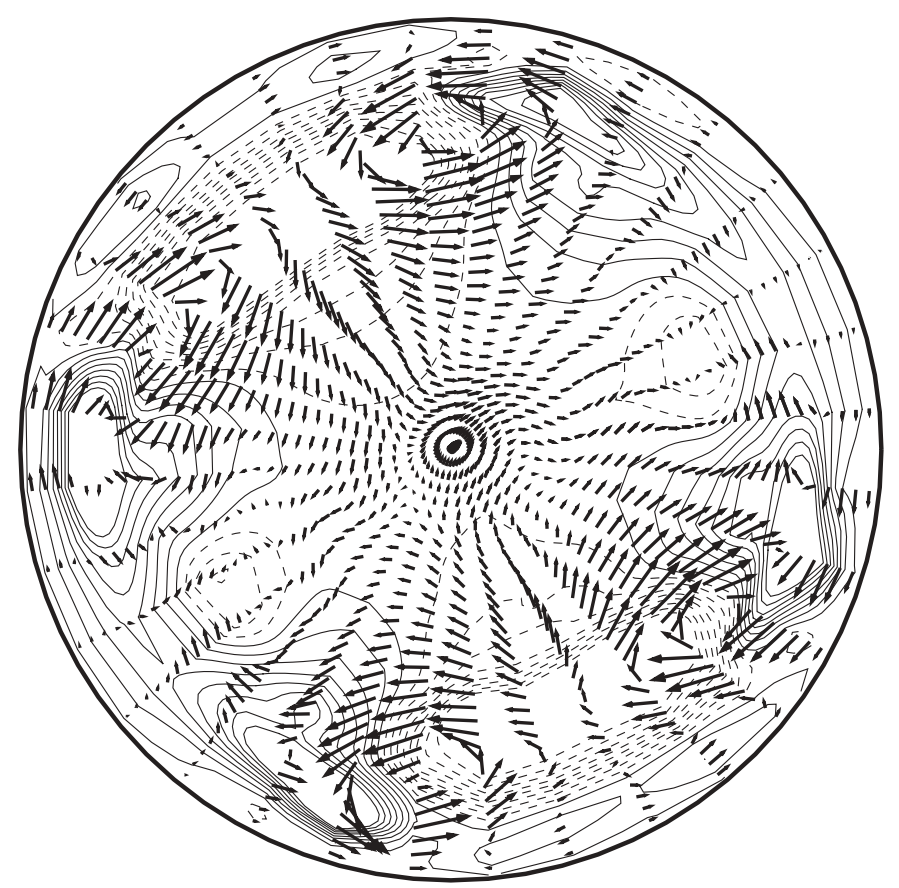

FIGURE 27. Instantaneous velocity field corresponding to the simulation shown in figures 20 and 21 at $x \simeq 56$. The contour lines range from -0.12 to 0.12 , spaced by 0.016 ; the vectors represent the secondary velocity in the pipe cross-section.

\section{Conclusions}

The present theoretical/computational investigation originates from experimental observations which highlight the considerable importance of base-flow distortions in the transition process of cylindrical pipe flow (e.g. Eliahou et al. 1998). Against this background, we have explored the spatial growth of infinitesimal disturbances developing on top of a distorted axially invariant pipe flow, which consists of the parabolic Hagen-Poiseuille modified by a small axisymmetric distortion. In actual experiments, such deviations from the theoretical velocity distribution may occur because of surface roughness, external volume forces, flow inhomogeneities, and so on. The limitation of the present study is that only axisymmetric axially invariant base-flow deformations have been considered.

To investigate the sensitivity to these base-flow variations, we have analysed the eigenvalues of the linearized Navier-Stokes operator and have identified the most sensitive eigenmodes. They are found to coincide with the eigenvalues recognised by Tumin (1996) as the most 'receptive' to periodic blowing and suction through a slot at the wall. With these 'sensitive' eigenvalues as point of departure, we have computed by means of a variational technique the so-called optimal base flow deviations for Hagen-Poiseuille flow, i.e. those distortions which maximally destabilize the flow. As a result, we have found that base-flow deviations of very small norm are capable of supporting perturbations, which show exponential growth. These so-called optimal deviations lead to inflection points in the base-flow profile suggesting an inviscid-type instability.

With the help of the same variational technique, we have then computed the dependence of the instability to parameters such as the Reynolds number, the 
azimuthal wavenumber and the frequency of the disturbances. The neutral curves, obtained for fixed values of the norm of the optimal base flow deviation $\epsilon$, show that streamwise waves of shorter wavelength and higher frequencies are excited as $\epsilon$ is decreased. If an experimentalist engaged in pipe-flow measurements could provide an estimate of the expected variance of the deviation on his axial velocity, results such as those provided in figures $13(a)$ and $14(a)$ would indicate whether or not exponential growth of infinitesimal perturbations could be expected in the experiments.

Further conclusions of the theory are that the mode $m=1$ is the most prone to destabilization by the mechanism described here and that the axisymmetric mode $m=0$ can also be excited at Reynolds numbers of the order of 2000 . In order to verify these theoretical results, the two modes with $m=1$ and $m=0$ have been used as initial conditions in direct numerical simulations to study possible transition paths. It is first noted that transition can be started by the amplification of either mode, provided its initial amplitude is sufficiently large and/or the base-flow distortion is strong enough. For the case with $m=1$, transition is observed even when the distortion is allowed to diffuse immediately from $x=0$ under the action of viscosity, while for the case with $m=0$, the base-flow distortion must be present for a finite length of the pipe.

The numerical simulations show also that the path of transition resulting from the growth of the axisymmetric mode is reminiscent of the subharmonic $H$-transition scenario of boundary layers, with the formation of staggered patterns of $\Lambda$-vortices. On the other hand, for the mode $m=1$, the formation of streamwise elongated streaks is observed, that break down through a secondary subharmonic instability. This latter scenario seems to be the counterpart of the oblique transition scenario of Berlin et al. (1994).

This paper has added but a brick to the building of a thorough comprehension of pipe-flow transition, by concentrating on plausible effects that can occur when the velocity distribution used as a reference in the initial linearization of the equations is not exactly equal to the theoretical profile. Future work will be devoted to the study of non-axisymmetric base-flow distortions, and to the optimal and robust control of pipe-flow transition.

The authors wish to thank Dr B.J. Boersma and Dr M. Pourquie, for their help with the numerical simulations. M.I.G. initiated this work in Toulouse with the support of the EU under a Marie Curie Training Site Fellowship, contract number HPMT-CT-2000-00079.

Appendix A. Non-zero elements of matrices $\mathscr{C}_{0}, \mathscr{C}_{1}$ and $\mathscr{C}_{2}$

$$
\begin{aligned}
\mathscr{C}_{0}^{12} & =1 \\
\mathscr{C}_{0}^{21} & =\frac{\left(m^{2}+1\right)}{r^{2}}-\mathrm{i} \omega \operatorname{Re} ; \mathscr{C}_{0}^{22}=\operatorname{Re} U(r) ; \mathscr{C}_{0}^{25}=\frac{2 \mathrm{i} m}{r^{2}}, \\
\mathscr{C}_{0}^{31} & =-\frac{1}{r} ; \mathscr{C}_{0}^{35}=-\frac{\mathrm{i} m}{r}, \\
\mathscr{C}_{0}^{41} & =\frac{U(r)}{r}-\frac{\mathrm{d} U}{\mathrm{~d} r} ; \mathscr{C}_{0}^{42}=-\frac{1}{\operatorname{Rer}} ; \mathscr{C}_{0}^{43}=\mathrm{i} \omega-\frac{m^{2}}{\operatorname{Rer}^{2}} ; \mathscr{C}_{0}^{45}=\frac{\mathrm{i} m U(r)}{r}, \\
\mathscr{C}_{0}^{46} & =-\frac{\mathrm{i} m}{\operatorname{Rer}},
\end{aligned}
$$




$$
\begin{aligned}
& \mathscr{C}_{0}^{56}=1, \\
& \mathscr{C}_{0}^{61}=-\frac{2 \mathrm{i} m}{r^{2}} ; \mathscr{C}_{0}^{64}=\frac{\mathrm{i} m R e}{r} ; \mathscr{C}_{0}^{65}=-\mathrm{i} \omega \operatorname{Re}+\frac{\left(m^{2}+1\right)}{r^{2}} ; \mathscr{C}_{0}^{66}=\operatorname{Re} U(r), \\
& \mathscr{C}_{1}^{21}=-\frac{1}{r} ; \mathscr{C}_{1}^{24}=\operatorname{Re}, \\
& \mathscr{C}_{1}^{31}=-1, \\
& \mathscr{C}_{1}^{41}=U(r) ; \mathscr{C}_{1}^{42}=-\frac{1}{\operatorname{Re}} ; \mathscr{C}_{1}^{43}=\frac{1}{\operatorname{Rer}}, \\
& \mathscr{C}_{1}^{65}=-\frac{1}{r}, \\
& \mathscr{C}_{2}^{21}=-1 ; \mathscr{C}_{2}^{43}=\frac{1}{\operatorname{Re}} ; \mathscr{C}_{2}^{65}=-1 .
\end{aligned}
$$

\section{Appendix B. Generalized Rayleigh and Fjørtoft theorems for inviscid flows in a cylindrical pipe geometry}

In this Appendix, we shall briefly review the Rayleigh inflection point theorem and the Fjørtoft criterion in cylindrical geometry (see also Batchelor \& Gill 1962). We restrict ourselves to axisymmetric disturbances and to their temporal evolution in the inviscid case, with a base flow given by $U(r)$. The equation for the shape function $\Phi$ of the streamfunction $\Psi(x, r, t)=\Phi(r) \exp ^{\mathrm{i} \alpha(x-c t)}$ is given by:

$$
\left(\frac{\mathrm{d}^{2}}{\mathrm{~d} r^{2}}-\frac{1}{r} \frac{\mathrm{d}}{\mathrm{d} r}-\alpha^{2}\right) \Phi-r\left(\frac{U^{\prime}}{r}\right)^{\prime} \frac{\Phi}{U-c}=0,
$$

where we have supposed that the temporal growth rate $c_{i}>0$ so that $U-c \neq 0$. The boundary conditions are: $\Phi / r$ and $\Phi^{\prime} / r$ bounded as $r \rightarrow 0$, and $\Phi=0$ at $r=1$. It is useful to rewrite equation (B 1 ) as follows:

$$
\left[r \frac{\mathrm{d}}{\mathrm{d} r}\left(\frac{1}{r} \frac{\mathrm{d}}{\mathrm{d} r}\right)-\alpha^{2}\right] \Phi-r\left(\frac{U^{\prime}}{r}\right)^{\prime} \frac{\Phi}{U-c}=0 .
$$

Multiplying each term of this equation by the complex conjugate $\left(\Phi^{*} / r\right)$, and integrating between $r=0$ and $r=1$, we obtain:

$$
\left.\left[\frac{\Phi^{\prime}}{r}\right] \Phi^{*}\right|_{0} ^{1}-\int_{0}^{1} \frac{\Phi^{\prime} \Phi^{*^{\prime}}}{r} \mathrm{~d} r-\alpha^{2} \int_{0}^{1} \frac{\Phi \Phi^{*}}{r} \mathrm{~d} r-\int_{0}^{1}\left(\frac{U^{\prime}}{r}\right)^{\prime} \frac{\Phi \Phi^{*}}{U-c} \mathrm{~d} r=0 .
$$

The first term is equal to zero on account of the boundary conditions, so that (B 3) can be rewritten as:

$$
\int_{0}^{1}\left(\frac{U^{\prime}}{r}\right)^{\prime} \frac{|\Phi|^{2}}{U-c} \mathrm{~d} r=-\int_{0}^{1} \frac{1}{r}\left[\left|\Phi^{\prime}\right|^{2}+\alpha^{2}|\Phi|^{2}\right] \mathrm{d} r,
$$

with the right-hand side purely real. By taking the imaginary part of (B 4), we obtain,

$$
c_{i} \int_{0}^{1}\left(\frac{U^{\prime}}{r}\right)^{\prime} \frac{|\Phi|^{2}}{|U-c|^{2}} \mathrm{~d} r=0 .
$$

Since we have assumed that $c_{i}>0$, this integral can become zero only if $\left(U^{\prime} / r\right)^{\prime}$ changes sign at least once in the open interval $(0,1)$. Hence, the necessary condition 
for instability is:

$$
\left(\frac{U^{\prime}}{r}\right)^{\prime}=0
$$

which is a generalization to cylindrical coordinates of Rayleigh's inflection-point criterion. The quantity $U^{\prime} / r$ can be interpreted as the circulation, which should therefore have a maximum somewhere in the flow for instability to be possible.

A stronger criterion of inviscid instability is provided by the Fjørtoft theorem, which can be derived by taking the real part of (B 4). This leads to:

$$
\int_{0}^{1}\left(\frac{U^{\prime}}{r}\right)^{\prime} \frac{U-c_{r}}{|U-c|^{2}}|\Phi|^{2} \mathrm{~d} r=-\int_{0}^{1} \frac{1}{r}\left[\left|\Phi^{\prime}\right|^{2}+\alpha^{2}|\Phi|^{2}\right] \mathrm{d} r<0 .
$$

If we now add to the left-hand side the term:

$$
\left(c_{r}-U_{s}\right) \int_{0}^{1}\left(\frac{U^{\prime}}{r}\right)^{\prime} \frac{|\Phi|^{2}}{|U-c|^{2}} \mathrm{~d} r=0,
$$

where $U_{s}=U\left(r_{s}\right)$ is the velocity of the base flow calculated at the radial position where (B 5) is satisfied, we obtain,

$$
\int_{0}^{1}\left(\frac{U^{\prime}}{r}\right)^{\prime} \frac{U-U_{s}}{|U-c|^{2}}|\Phi|^{2} \mathrm{~d} r<0,
$$

from which it follows that a necessary condition for instability is:

$$
\left(\frac{U^{\prime}}{r}\right)^{\prime}\left(U-U_{s}\right)<0
$$

somewhere in the flow field. Although (B 5) and (B 7) are rather straightforward extensions of classical results for plane shear flows, we feel that it is useful to report them here since we have been unable to find (B 7) in the literature.

\section{REFERENCES}

Andersson, P., Brandt, L., Bottaro, A. \& Henningson, D. S. 2001 On the breakdown of boundary layer streaks. J. Fluid Mech. 428, 29-60.

Barkley, D. \& Tuckerman, L. S. 1999 Stability analysis of perturbed plane Couette flow. Phys. Fluids A 5, 1187-1195.

Batchelor, G. K. \& Gill, A. E. 1962 Analysis of the stability of axisymmetric jets. J. Fluid Mech. 14, 529-551.

BergströM, L. 1992 Initial algebraic growth of small angular dependent disturbances in pipe Poiseuille flow. Stud. Appl. Maths 87, 61-79.

Bergström, L. 1993 Optimal growth of small disturbances in pipe Poiseuille flow. Phys. Fluids A 5, 2710-2720.

Berlin, S., Lundbladh, A. \& Henningson, D. S. 1994 Spatial simulations of oblique transition in a boundary layer. Phys. Fluids 6, 1949-1951.

Berlin, S., Wiegel, M. \& Henningson, D. S. 1999 Numerical and experimental investigations of oblique boundary layer transition. J. Fluid Mech. 393, 23-57.

Boberg, L. \& Brosa, U. 1988 Onset of turbulence in a pipe. Z. Naturforsch. 43a, 697-726.

Bottaro, A., Corbett, P. \& Luchini, P. 2003 The effect of base flow variation on flow stability. J. Fluid Mech. 476, 293-302.

Bottin, S., Dauchot, O., Daviaud, F. \& Manneville, P. 1998 Experimental evidence of streamwise vortices as finite amplitude solutions in transitional plane Couette flow. Phys. Fluids 10, $2597-2607$. 
Clever, R. M. \& Busse, F. H. 1992 Three dimensional convection in a horizontal fluid layer subjected to a constant shear. J. Fluid Mech. 234, 511-527.

Darbyshire, A. G. \& Mullin, T. 1995 Transition to turbulence in constant mass-flux pipe flow. J. Fluid Mech. 289, 83-114.

DaveY, A. 1978 On the stability of flow in an elliptic pipe which is nearly circular. J. Fluid Mech. 87, 233-241.

Davey, A. \& Drazin, P. G. 1969 The stability of Poiseuille flow in a pipe. J. Fluid Mech. 36, 209-218.

Draad, A. A., Kuiken, G. D. C. \& Nieuwstadt, F. T. M. 1998 Laminar-turbulent transition in pipe flow for Newtonian and non-Newtonian fluids. J. Fluid Mech. 377, 267-312.

Eggels, J. G. M., Unger, F., Weiss, M. H., Westerweel, J., Adrian, R. J., Friedrich, R. \& Nieuwstadt, F. T. M. 1994 Fully developed turbulent pipe flow: a comparison between direct numerical simulation and experiment. J. Fluid Mech. 268, 175-209.

Eliahou, S., Tumin, A. \& Wygnanski, I. 1998 Laminar-turbulent transition in Poiseuille pipe flow subjected to periodic perturbation emanating from the wall. J. Fluid Mech. 361, 333-349.

Faisst, H. \& Eckhardt, B. 2003 Travelling waves in pipe flow. Phys. Rev. Lett. 91, 224502.

Garg, V. K. \& Rouleau, W. T. 1972 Linear spatial stability of pipe Poiseuille flow. J. Fluid Mech. 54, 113-127.

GiLl, A. E. 1965 A mechanism for instability of plane Couette flow and of Poiseuille flow in a pipe. J. Fluid Mech. 21, 503-511.

Han, G., Tumin, A. \& Wygnanski, I. 2000 Laminar-turbulent transition in Poiseuille pipe flow subjected to periodic perturbation emanating from the wall. Part 2. Late stage of transition. J. Fluid Mech. 419, 1-27.

Henningson, D. S., Lundbladh, A. \& Johansson, A. V. 1993 A mechanism for bypass transition from localized disturbances in wall-bounded shear flows. J. Fluid Mech. 250, 169-207.

Herron, I. 1991 Observations on the role of vorticity in the stability theory of wall bounded flows. Stud. Appl. Maths 85, 269-286.

Joseph, D. D. \& Carmi, S. 1969 Stability of Poiseuille flow in pipes, annuli and channels. Q. Appl. Maths 26, 575-591.

Kerswell, R. R. \& Davey, A. 1996 On the linear instability of elliptic pipe flow. J. Fluid Mech. 316, 307-324.

Leite, R. J. 1959 An experimental investigation of the stability of Poiseuille flow. J. Fluid Mech. 5, $81-96$.

Lessen, M., Sadler, S. G. \& Liu, T. Y. 1968 Stability of pipe Poiseuille flow. Phys. Fluids 11, $1404-1409$.

Lundbladh, A., Schmid, P. J., Berlin, S. \& Henningson, D. S. 1994 Simulations of bypass transition for spatially evolving disturbances. $A G A R D-C P-551$, pp. 18.1-18.13.

Ma, B., van Doorne, C. W. H., Zhang, Z. \& Nieuwstadt, F. T. M. 1999 On the spatial evolution of a wall-imposed periodic disturbance in pipe Poiseuille flow at $R e=3000$. Part 1. Subcritical disturbance. J. Fluid Mech. 398, 181-224.

Matsubara, M. \& Alfredsson, P. H. 2001 Disturbance growth in boundary layers subjected to free-stream turbulence. J. Fluid Mech. 430, 149-168.

Meseguer, A. \& Trefethen, L. N. 2003 Linearized pipe flow to Reynolds number $10^{7}$. J. Comput. Phys. 186, 178-197.

Morkovin, M. V. 1993 Bypass-transition research: issues and philosophy. In Instabilities and Turbulence in Engineering Flows (ed. D. E. Ashpis, T. B. Gatski \& R. Hirsh), pp. 3-30. Kluwer.

NaGATA, M. 1990 Three-dimensional finite-amplitude solutions in plane Couette flow: bifurcation from infinity. J. Fluid Mech. 217, 519-527.

O’Sullivan, P. L. \& Breuer, K. S. 1994a Transient growth in circular pipe flow. Part I: Linear disturbances. Phys. Fluids 6, 3643-3651.

O'Sullivan, P. L. \& BreueR, K. S. $1994 b$ Transient growth in circular pipe flow. Part II: Nonlinear development. Phys. Fluids 6, 3652-3664.

Pfenniger, W. 1961 Boundary layer suction experiments with laminar flow at high Reynolds numbers in the inlet of a tube by various suction methods. In Boundary Layer and Flow Control. Its Principles and Applications (ed. G. V. Reynolds), vol. 2, pp. 961-980. Pergamon. 
Reuter, J. \& Rempfer, D. 2000 High order vorticity-velocity method for the simulation of pipe flow transition. Appl. Num. Maths 33, 105-111.

REYNOLDS, O. 1883 An experimental investigation of the circumstances which determine whether motion of water shall be direct or sinuous and of the law of resistance in parallel channels. Phil. Trans. R. Soc. Lond. 174, 935-994.

Salwen, H., Cotton, F. W. \& Grosch, C. E. 1980 Linear stability of Poiseuille flow in a circular pipe. J. Fluid Mech. 98, 273-284.

Schmid, P. J. \& Henningson, D. S. 1994 Optimal energy density growth in Hagen-Poiseuille flow. J. Fluid Mech. 277, 197-225.

Schmid, P. J. \& Henningson, D. S. 2001 Stability and Transition in Shear Flows. Springer.

Trefethen, L. N., Chapman, S. J., Henningson, D. S., Meseguer, A., Mullin, T. \& Nieuwstadt, F. T. M. 2000 Threshold amplitudes for transition to turbulence in a pipe. Oxford University, Numerical Analysis Group, Rep. 00/17.

Trefethen, L. N., Trefethen, A. E., Reddy, S. C. \& Driscoll, T. A. 1993 Hydrodynamic stability without eigenvalues. Science 261, 578-584.

Tumin, A. 1996 Receptivity of pipe Poiseuille flow. J. Fluid Mech. 315, 119-137. 